\title{
Electron-cloud simulation and theory for high-current heavy-ion beams
}

\author{
R. H. Cohen, ${ }^{1, *}$ A. Friedman, ${ }^{1}$ S. M. Lund, ${ }^{1}$ A. W. Molvik, ${ }^{1}$ E. P. Lee, ${ }^{1}$ T. Azevedo, ${ }^{2}$ J.-L. Vay, ${ }^{2}$ P. Stoltz, ${ }^{3}$ and S. Veitzer ${ }^{3}$ \\ ${ }^{1}$ Lawrence Livermore National Laboratory, P.O. Box 808, Livermore California 94550, USA \\ ${ }^{2}$ Lawrence Berkeley National Laboratory, Berkeley, California 94720, USA \\ ${ }^{3}$ TechX Corporation, 5621 Arapahoe Avenue, Suite A, Boulder, Colorado 80303, USA
}

(Received 30 July 2004; published 8 December 2004)

\begin{abstract}
Stray electrons can arise in positive-ion accelerators for heavy-ion fusion or other applications as a result of ionization of ambient gas or gas released from walls due to halo-ion impact, or as a result of secondary-electron emission. We summarize the distinguishing features of electron-cloud issues in heavy-ion-fusion accelerators and a plan for developing a self-consistent simulation capability for heavy-ion beams and electron clouds (also applicable to other accelerators). We also present results from several ingredients in this capability. (1) We calculate the electron cloud produced by electron desorption from computed beam-ion loss, which illustrates the importance of retaining ion reflection at the walls. (2) We simulate the effect of specified electron-cloud distributions on ion beam dynamics. We consider here electron distributions with axially varying density, centroid location, or radial shape, and examine both random and sinusoidally varying perturbations. We find that amplitude variations are most effective in spoiling ion beam quality, though for sinusoidal variations which match the natural ion beam centroid oscillation or breathing-mode frequencies, the centroid and shape perturbations can also have significant impact. We identify an instability associated with a resonance between the beamenvelope "breathing" mode and the electron perturbation. We estimate its growth rate, which is moderate (compared to the reciprocal of a typical pulse duration). One conclusion from this study is that heavy-ion beams are surprisingly robust to electron clouds, compared to a priori expectations. (3) We report first results from a long-time-step algorithm for electron dynamics, which holds promise for efficient simultaneous solution of electron and ion dynamics.
\end{abstract}

DOI: 10.1103/PhysRevSTAB.7.124201

PACS numbers: 52.59.Sa, 52.59.Fn, 52.65.Rr, 52.65.Cc

\section{INTRODUCTION}

Heavy-ion fusion (HIF) entails the acceleration of beams of heavy ions to drive inertially confined fusion targets. The mainline approach in the U.S. envisions the use of induction linear accelerators to accelerate multiple beams of ions from an energy of the order of $1 \mathrm{MeV}$ and a current of order of an ampere per beam, with a pulse duration (time to pass a stationary observer) of 10's of $\mu \mathrm{s}$, to an energy of the order of few $\mathrm{GeV}$, a current of order of $1 \mathrm{kA} /$ beam, and a duration of $\sim 10 \mathrm{~ns}$.

Like other positive-charge-particle accelerators, an accelerator for HIF is subject to contamination by stray electrons, which can be electrostatically trapped by the ion beam potential. This is a phenomenon that has been documented in a range of positive-charge-particle accelerators dating back to the 1960's [1]; see Refs. [2,3] and references therein. The common concern is that the electron cloud is an uncontrolled negative charge that can alter the ion beam dynamics, possibly leading to beam deflection, increased beam emittance, envelope size, and halo, and also potentially electron-ion instabilities. On the other hand, HIF has a number of distinguishing features that impact both the nature and the modeling of electron clouds.

\footnotetext{
*Electronic address: rcohen@1lnl.gov
}

In this paper we survey the distinguishing features of HIF for electron clouds, discuss an outline of a selfconsistent simulation capability for ion beams in the presence of electron clouds, based on the WARP particle simulation code [4], and present results from two simulation studies: the electron cloud produced by electron desorption from ion beam loss, and the effect of specified electron clouds on ion beam dynamics. In the course of presentation of the latter study, we identify an instability associated with ion beam loss. We also briefly present first results from a long-time-step algorithm for electrons which may greatly facilitate simultaneous electron and ion simulation; this algorithm will be presented in more detail in a separate paper.

The remainder of this paper is organized as follows: Sec. II is a summary of the relevant distinguishing features of heavy-ion induction accelerators, their consequences for electron-cloud physics, and our plan for self-consistent electron-cloud simulation. In Sec. III we present results for the electron cloud obtained from a chain of calculations that represent a number of the ingredients in our plan. Section IV is devoted to our studies of the effect of prescribed electron-cloud perturbations on ion beam quality. Section V discusses a beamloss instability suggested by the results of Sec. IV, and develops an estimate of the (self-consistent) growth rate based on the results from the (non-self-consistent) study of Sec. IV. Section VI revisits the electron-cloud simula- 
tion of Sec. III, but with the long-time-step algorithm mentioned above. Section VII is a summary and discussion of our results.

It should be noted at this point that, while the simulation tools and road map described in this paper were developed with the distinguishing features of HIF accelerators in mind, and the applications described are HIF motivated, the tools themselves have applicability to a broad range of positive-charged-particle accelerators. With the addition of photoemission, the plan discussed in Sec. II (and being implemented in the WARP code) would be applicable to any of the accelerator configurations for which electron-cloud issues have been raised in recent conferences $[2,3]$.

\section{DISTINGUISHING FEATURES OF HIF INDUCTION ACCELERATORS}

Induction accelerators for heavy-ion fusion have a number of features that distinguish them from accelerators for other applications, such as high-energy physics. We summarize here those that are relevant to electroncloud issues. We refer here to the mainline approach in the U.S., which is based on induction linacs with magnetic quadrupole focusing.

These accelerators are linear machines, with a high line-charge density and perveance (high enough that space charge dominates over emittance as the principal defocusing mechanism). The pulses are rather longmulti- $\mu \mathrm{s}$ at the injector end, compressed to a fraction of a $\mu \mathrm{s}$ at the high-energy end, and then compressed to a duration on the order of $10 \mathrm{~ns}$ before entering the target chamber. As a consequence of these features, the electron transit time across a beam pipe is small compared to the beam transit time. Hence the electron multipactor process common in short-pulse circular machines, where the electron transit time resonates with the pulse repetition time, is not an issue. On the other hand, a long-pulse multipactor process is possible on the trailing edge of the beam: electrons transiting the beam gain energy due to the decreasing beam potential, possibly leading to energies where the secondary-emission coefficient exceeds unity and therefore exponentiation of the electron density. HIF beams are intended to have a long flattop. Hence multipactoring, if it occurs, should be confined to the short tail section.

Another important distinguishing feature is that there is an economic mandate to maximally fill the beam pipe. (It is desired that the peak semimajor axis of the elliptical beam be $60 \%-80 \%$ of the pipe radius). Hence, any unanticipated defocusing effects (for example, from an electron cloud, or from magnet misalignment) will lead to beam scrape-off, and the electrons resulting from direct emission or from ionization of desorbed gas become a concern. Indeed, electron-cloud effects may set the limit for beam fill.
HIF induction accelerators have a relatively large fraction of their length occupied by quadrupole magnets $50 \%$ or more at the injector end. For this reason (and because a dominant source of electrons is localized there), electron dynamics in the quadrupoles plays a major role.

Heavy-ion accelerators have a large neutral desorption coefficient-typically of order $10^{4}$ per lost ion $[5,6]$. Particularly for the long pulses at the injector end of an accelerator, this can make ionization of desorbed neutral gas the dominant source of electrons.

Induction accelerators have beam pipes in the magnet sections and not in the accelerating gaps in between; also the varying ellipticity of the beam is maximum within the magnets. Hence desorption is confined to the quadrupoles. The beam space charge potential is typically large (a few $\mathrm{kV}$ at the injector end) compared to the birth energy of electrons from ionization or desorption, but smaller than the potential across an accelerating gap. Consequently, most electrons born from ionization of desorbed gas are magnetically confined against motion in the beam direction and trapped by a deep potential well and magnetic mirror forces in the transverse plane. (The exceptions are the very few electrons born right at the wall or very close to the magnetic field null.) These electrons bounce transversely and slowly drift under the combined influence of magnetic and electric drifts in the beamwise (or counterbeamwise) direction. In the plane transverse to the beam, the electrons tend to be concentrated within the beam cross section, since that is where they are born and the beam potential is attractive for them.

The drift time $\tau_{d} \sim L_{q} /\left(v_{E}+v_{B}\right)$, where $L_{q}$ is the quadrupole length, $v_{E}$ is the $\mathbf{E} \times \mathbf{B}$ drift velocity, and $v_{B}$ is the magnetic ( $\nabla B$ and curvature) drift velocity, is typically a fraction of the (fixed-station) pulse duration, for characteristic parameters such as those listed in Appendix A. Hence, before the pulse is over, electrons can drift out of a quadrupole. Electrons in two quadrants of the quadrupole will exit backwards (relative to the beam propagation) into an accelerating gap, and be accelerated sufficiently to escape the beam potential. These electrons will tend to get lost upon encountering the fringe field of the next quadrupole. Electrons in the remaining two quadrants exit forward into an accelerating gap, and then bounce longitudinally between the quadrupole and the (decelerating) gap field, until they have moved transversely into a quadrupole field quadrant where the drift is back into the quadrupole. These electrons then drift back through the quad and get lost after traversing the next gap. The net result is that the lifetime for all electrons is of the order of the time to drift through a quadrupole. This succession of events is illustrated by the orbit calculation shown in Fig. 1. A consequence of these processes is that the electron density will be largely 


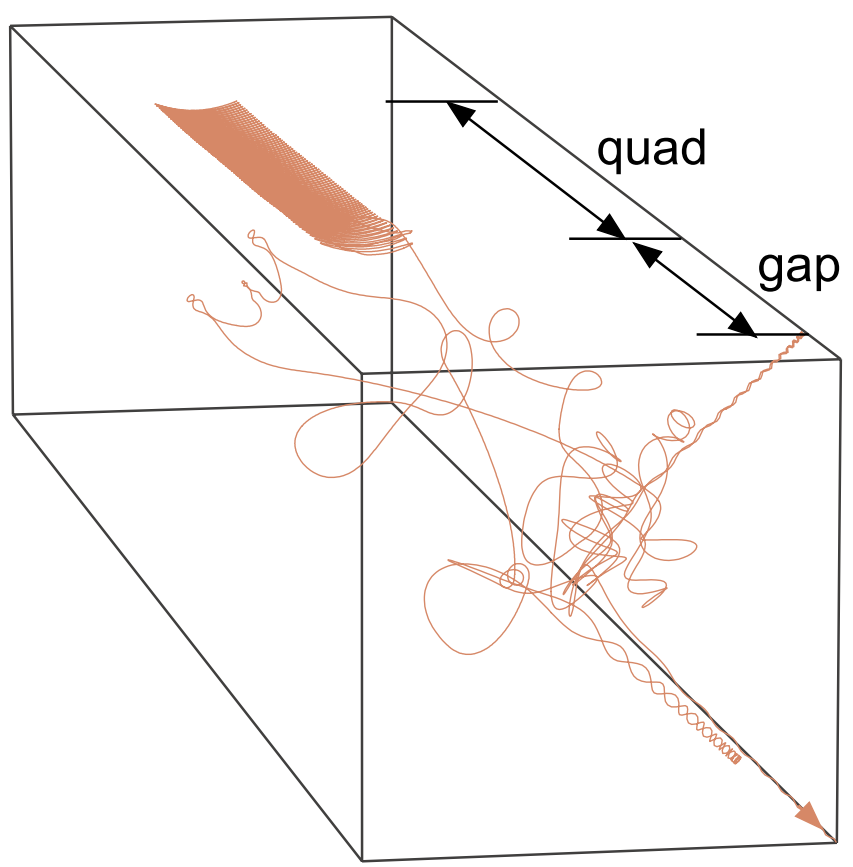

FIG. 1. (Color) Orbit of test electron initialized in a quadrupole magnet. Electron is initially magnetically and electrostatically trapped, drifts into an accelerating gap, is accelerated (backwards from beam), and escapes in fringe field of next quadrupole.

confined to the quadrupoles, and be larger, by about a factor of 2, in the quadrants where the drift is counter to the beam direction. Also the electron density in a quadrupole will depend mainly on the sources in that quadrupole.

For a transport section (no acceleration in the gaps), the same confinement and drift processes described above will lead to a random walk of electrons from magnetic quadrupole to magnetic quadrupole. Since the time to drift out of a quadrupole $\tau_{d}$ is long compared to the time to transit a gap (at the electron thermal speed), the electron lifetime is of the order of the time to random walk out of the quadrupole system, $\tau \sim N_{q}^{2} \tau_{d}$, where $N_{q}$ is the number of quadrupoles, or the pulse duration (whichever is shorter). In the absence of sinks in the quadrupoles, the density contrast between quadrupoles and gaps will not be as large, and sources in one quadrupole impact the density in its neighbors. Since, for times of interest, buildup from ionization of desorbed gas is not a steady-state process $\left(n_{e} \propto t^{2}\right.$ for times $\left.t \ll \tau\right)$, there is a surviving density contrast and quadrupole-to-quadrupole independence which requires solution of the timedependent axial transport problem to quantify.

For shorter pulses (for example, at the high-energy end of the accelerator), desorbed neutral gas does not have time to penetrate deep into the beam interior, in which case electron desorption at the wall can become the dominant source. We take, as the criterion for "shorter," that an average-speed neutral not have time to travel halfway from the wall to the pipe center, i.e., $\tau_{\text {pulse }} \ll$ $r_{p} / 2 v_{n}$, where $r_{p}$ is the pipe radius and $v_{n}$ is a typical neutral speed; for $r_{p} \sim 3 \mathrm{~cm}$ and $\sim 1 \mathrm{eV}$ atomic hydrogen (as one might expect for electronic sputtering), this becomes $\tau_{\text {pulse }} \ll 1.5 \mu \mathrm{s}$. Because the elliptical distortion of the beam envelope in the quadrupole focusing system results in scrape-off primarily at locations which are axially well within a quadrupole magnet, but at wall locations where magnetic field lines are nearly tangent to the walls (see Fig. 2), the resulting electron cloud will be concentrated mainly at the edge, as will be seen in the calculations in the next section. The nominal lifetime for such electrons is one transit during the beam flattop, as they are born at the wall with some finite speed along the field line, and so can reach the wall at the opposite end of the field line. But secondary electron production, trapping due to nonadiabatic pitch-angle scattering [7] or due to interaction with fluctuations in the beam potential could increase this lifetime (with the nonadiabatic scattering being particularly effective for the few electrons born on field lines that penetrate deep into the beam center). Desorbed neutral gas is also concentrated in the same regions for this short-pulse case, and hence electrons produced from its ionization will have a spatial distribution resembling that from direct electron desorption; but these electrons are born trapped in the combined electrostatic and magnetic wells of the beam and the magnet.

We conclude this section by noting that a quantitative description of the electron cloud in an HIF accelerator and/or its effects on the ion beam requires a selfconsistent (simultaneous) calculation of the electron and

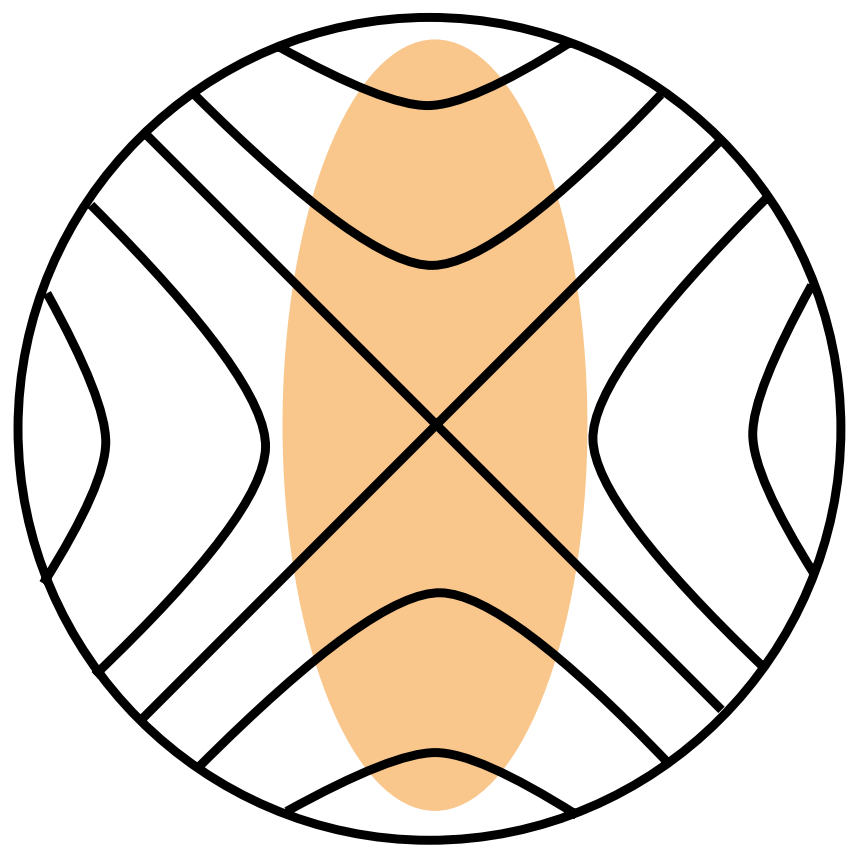

FIG. 2. (Color) Sketch of magnetic field lines and elliptical beam envelope in a quadrupole magnet. 
ion dynamics. Because beam scrape-off is a significant (dominant) electron source and because of the long pulse, electron-ion interaction affects the electron source. This is not the case for short-pulse, small fill-factor accelerators (e.g., for high-energy physics) where ionization of background ambient gas and photoelectron production, followed by electron multipactoring, are responsible for electron cloud buildup; in such a case a sequential chain of calculations may suffice: calculate the primary electron sources; calculate buildup from multipactoring and distribution from electron dynamics; calculate the impact of this time-evolving cloud on the ion beam.

The fully self-consistent simulation required for HIF accelerators is challenging because of the need to deal with electrons both in the quadrupole magnets and between quadrupoles. In the combined magnetic and beam-potential fields, one cannot analytically integrate electron orbits, and hence one must be able to deal with time scales ranging from the electron-cyclotron period $\left(10^{-10}-10^{-11} \mathrm{~s}\right)$ through the ion beam transit time $\left(10^{-5}-10^{-7} \mathrm{~s}\right)$. The multiplicity of electron sources is also a complication.

One approach to addressing this self-consistent modeling challenge is through a coupled set of simulation modules, as depicted in Fig. 3. This is the approach being taken in extending the WARP code. The preexisting WARP code solved for the self-consistent beam-ion distribution function and beam potential. The beam distribution function at the wall becomes input for calculations of desorbed electrons and gas [8], and reflected ions. The gas module must track desorbed gas and that from the ambient background, in the presence of charge exchange and ionization events (which in turn depend on the volumetric beam distribution). The wall electron source includes electron desorption and secondary emission, and to this must be added the volumetric source from ionization. The subsequent electron dynamics must be followed (including sinks at bounding surfaces), and its charge density deposited on the simulation grid and fed back into the ion simulations. In order to simultaneously simulate electrons and ions without the huge computational burden of time stepping on the electron-cyclotron time scale, we have developed a scheme to interpolate between full electron dynamics and the drift approximation; this will be described in Sec. VI and in more detail elsewhere. The current status of the various modules is indicated by the color coding in this figure.

\section{ELECTRON-CLOUD ACCUMULATION SIMULATIONS}

As an example of our current capability, we show calculations of the electron cloud resulting from desorption of electrons when primary and secondary (scattered) ions strike the walls. The simulation was done for a 100 lattice-period (200 quadrupoles) transport system, with parameters similar to compact magnetics designed for future application in the High-Current Experiment (HCX) [9] at Lawrence Berkeley National Laboratory. Each magnet is identical, and represented by gridded field data from a 3D magnet calculation. The magnets are $10.2 \mathrm{~cm}$ long and have a radial field gradient of $90.51 \mathrm{~T} / \mathrm{m}$ at the axial midplane of the magnet. The gaps between focusing and defocusing quadrupoles alternate in length between 6.1 and $18.5 \mathrm{~cm}$ ("syncopated doublet" lattice), giving a total lattice period of $45 \mathrm{~cm}$. The beam is taken to be bounded by a circular-crosssection beam pipe, with radius $2.95 \mathrm{~cm}$. Simulation particles that reach the pipe wall are removed from the computation or scattered as described below. A list of

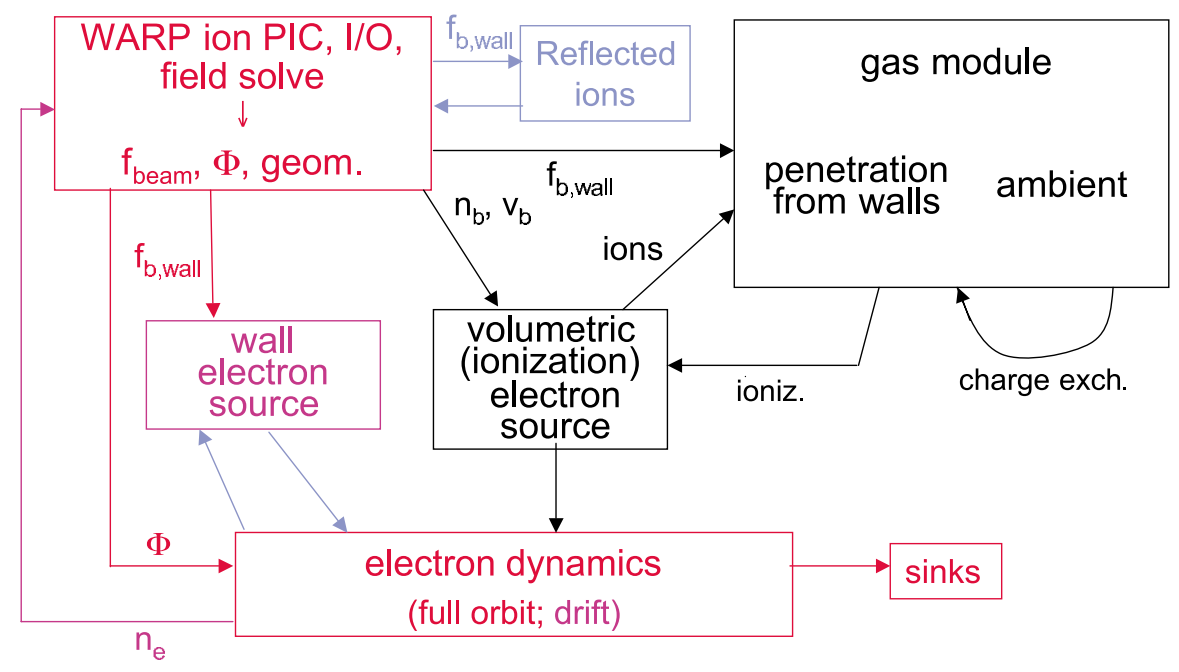

FIG. 3. (Color) Plan for self-consistent modeling of electron cloud and ion beam. Modules in red are presently operational in wARP code; modules in magenta are implemented and being tested; modules in blue are partially implemented; modules in black are being developed off-line. 
beam, lattice, and simulation parameters is given in Appendix A.

This simulation was done through a sequence of calculations, as follows. First, a WARP "slice" simulation [following 400000 ions in a two-dimensional transverse slice (in $x, y$ ) of the ion beam through the lattice] was done for a $2 \mathrm{MeV}$ potassium beam. The magnets were deliberately slightly misaligned (random transverse displacements of $500 \mu \mathrm{m}$ ) to exaggerate beam halo scrapeoff. Data were gathered for beam ions impacting the wall (6282 ions). The TRIM surface Monte Carlo code [10] was run to generate a population of 3629 scattered ions. The scattered ions were inserted into a three-dimensional WARP simulation and followed until their next wall impact. For both the primary and secondary ion impacts, the number of electrons desorbed was calculated using a fit to the experimental data from Ref. [6], $n_{e}\left(\approx u^{2} /\left\langle u^{2}\right\rangle\right) \times$ $\min (6 / \cos \phi, 130)$, where $\left\langle u^{2}\right\rangle$ is the average of the square of the velocity of the lost primary ions, $u$ is the speed of the lost ion under consideration, and $\phi$ is the angle between the normal to the surface and the velocity of the incident ion. A single particle with a weight given by the above expression is then introduced into WARP. The speed distribution is taken as uniform out to $8 \mathrm{eV}$, and the angular distribution goes as $\cos (\theta)$, where $\theta$ is the angle between the normal to the surface and the velocity of the emitted electron. The resulting electron population is followed in WARP (3D) for 4000 time steps, where the time step is chosen to be $1 / 4$ the cyclotron period at the wall, enough for several electron bounces. Electrons that reach the wall are considered as lost and removed from the calculation. The time-integrated electron charge density is formed by incrementally depositing the weighted electron charge density onto the grid.

The three-dimensional results for the electron density, projected on to a two-dimensional plane transverse to the beam, are shown in Fig. 4 keeping only the electrons from the primary (beam) ion loss, and in Fig. 5 keeping the electrons desorbed from loss of both the primary and scattered ions. We see that, without the scattered ions, virtually all of the electron density is located at the edge near the $0,90,180$, and 270 degree points. This is because all of the scrape-off of ions occurs near the tips of the elliptical beam envelope midway through each quadrupole magnet (see Fig. 2). If we add ion scattering, some ions scatter so as to next impact the wall at field lines which penetrate deep into the interior, as sketched in Fig. 6. A scattered-ion angular distribution from TRIM is shown in Fig. 7. While the distribution peaks for scattering in the plane formed by the incident ion trajectory and the wall, there is a small but significant tail of scattering well out of this plane. Keeping the electrons produced by scrape-off of this tail results in the electron cloud shown in Fig. 5. While the electron density is still peaked near the wall at the four azimuthal angles noted above, there is now a significant density everywhere.

We have recalculated the electron density using the long-time-step interpolated electron mover mentioned above and obtained almost identical results, which will be presented, along with a brief description of the mover, in Sec. VI.

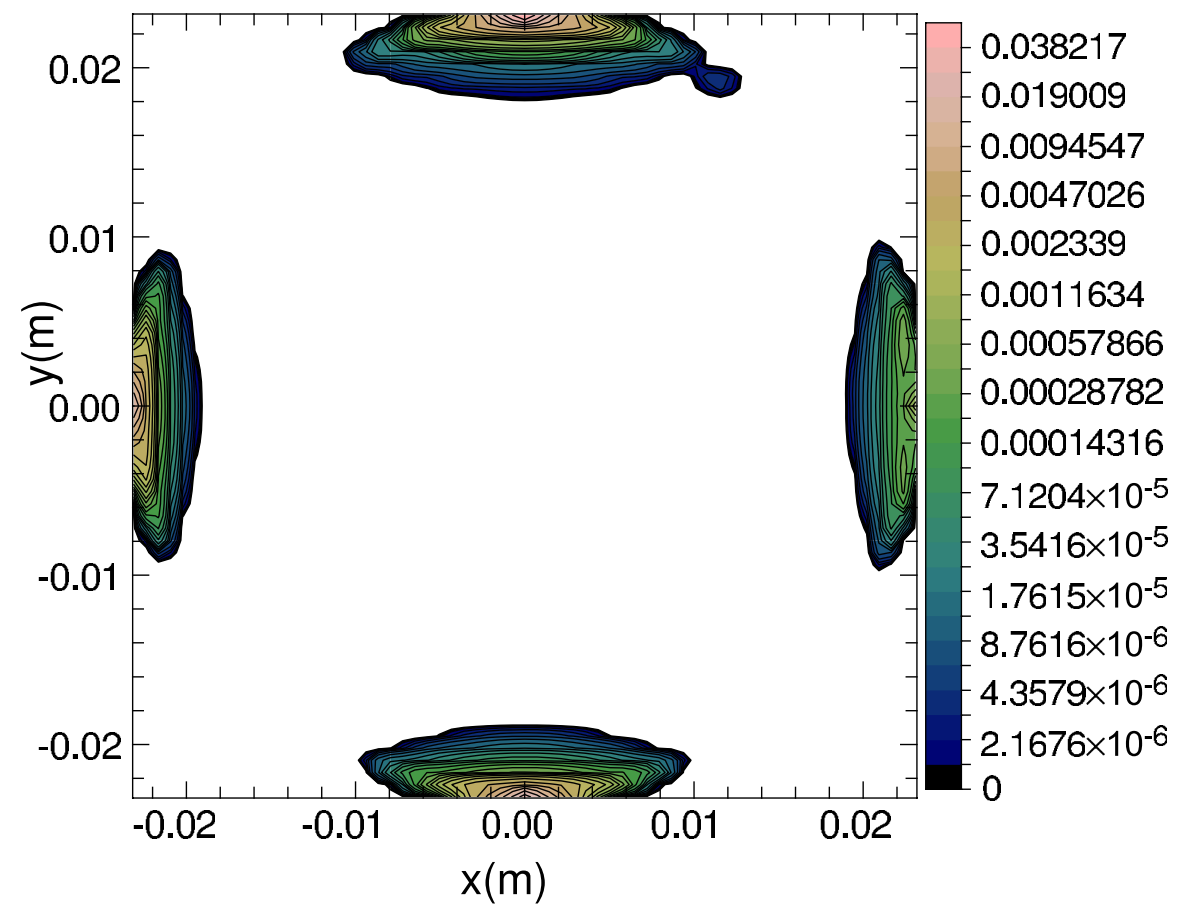

FIG. 4. (Color) $x-y$ projection of electron cloud density retaining only electrons desorbed by primary beam-ion impact at walls. 


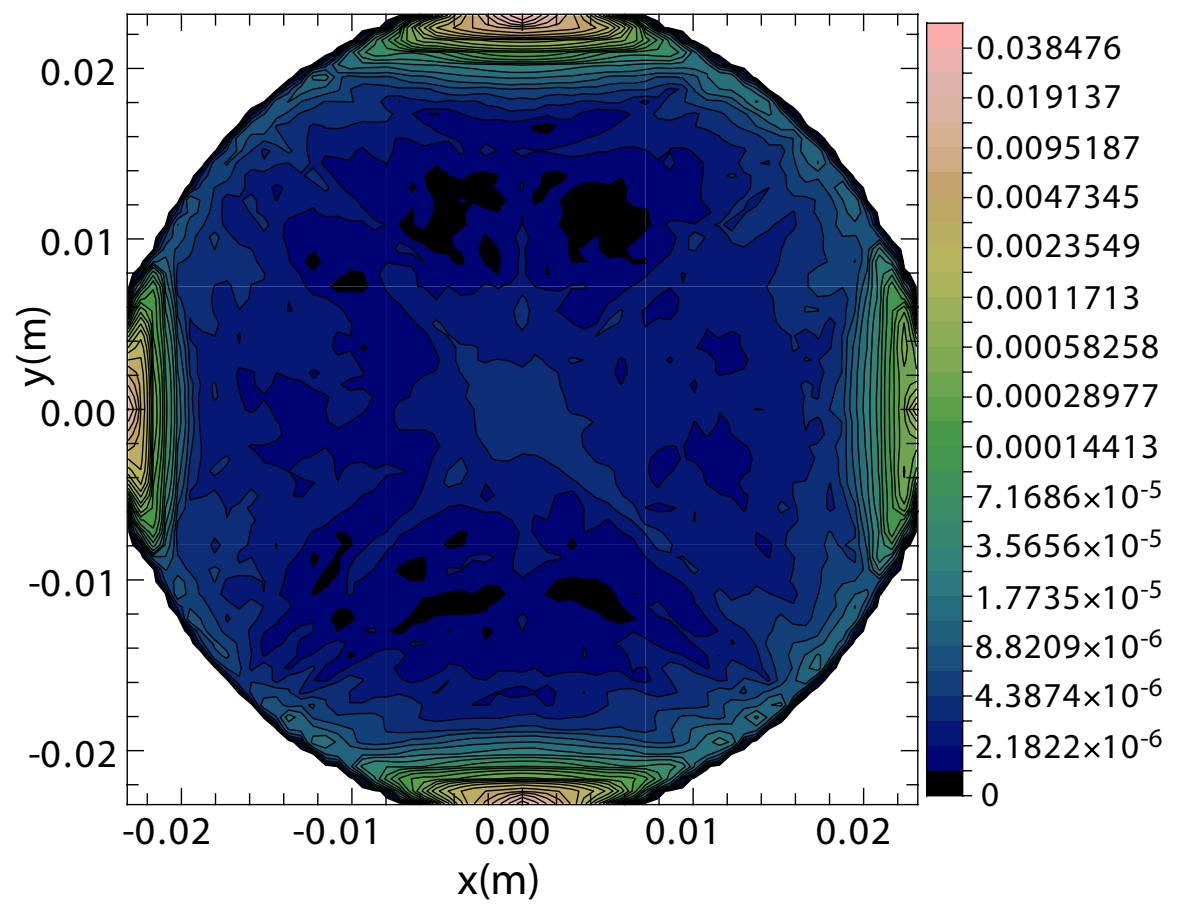

FIG. 5. (Color) $x-y$ projection of electron cloud density retaining electrons desorbed by impact of primary beam and scattered ions at walls.

It is interesting that there is a small local maximum near the center of the pipe. We believe this feature is not a statistical fluctuation, as it has occurred each time we have run the problem (as our scattered-ion model and our

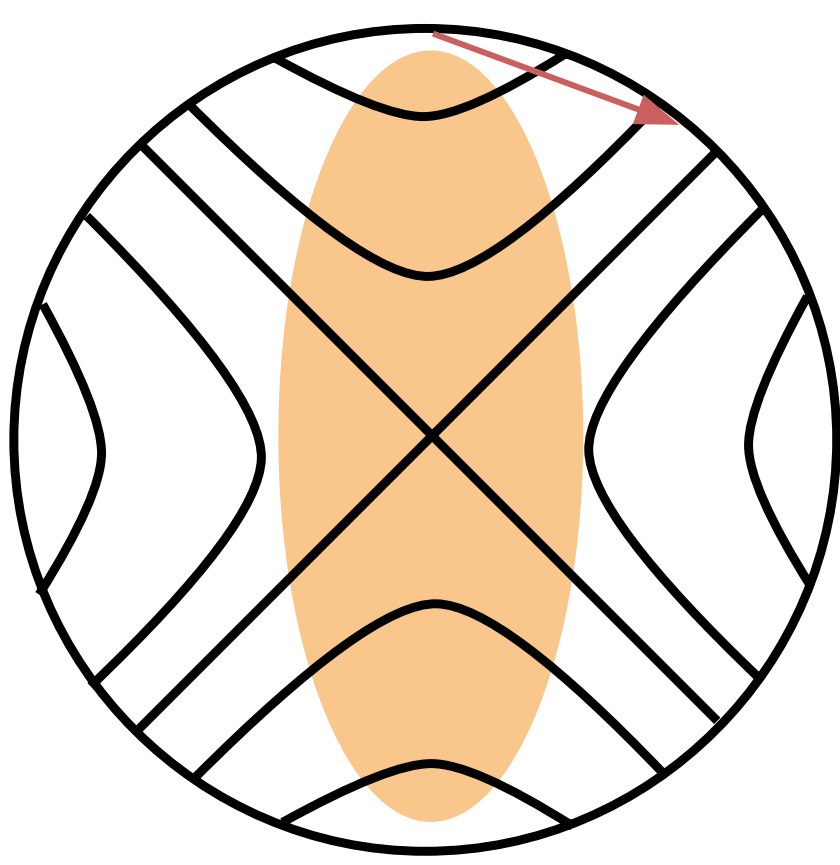

FIG. 6. (Color) Sketch of magnetic field lines and elliptical beam envelope in a quadrupole magnet; the arrow depicts scattering of primary beam ion. beam scrape-off diagnostic have both evolved). It also occurs for both our small-time-step and interpolated electron movers. It is contrary to what one would expect for electrons born at the wall and strictly following magnetic field lines which spread (defocusing the electrons) as they approach the center. We tentatively attribute this to nonadiabatic scattering [7], which preferentially affects electrons that pass close to the center and can transiently trap electrons in the magnetic well.

\section{EFFECT OF MODEL ELECTRON CLOUDS ON ION DYNAMICS}

We describe in this section a set of studies consisting of WARP slice simulations in which we have added a specified, frozen-in-time, negative charge distribution which represents the electron cloud. Such a model has the obvious deficiencies of being non-self-consistent and ignoring all electron dynamics. Nevertheless, it is useful for providing an indicator of the sensitivity of ion beams to the presence of electrons and hence estimates of tolerable electron levels, and also for illuminating important electron-ion interaction mechanisms, as will be seen.

A fixed-charge model is in some respects equivalent to modifying the external focusing of the beam. But this analogy is of limited utility. We will be exploring the effects of various spatial variations in the electron-cloud distribution. In all cases the model electron cloud is of finite radial extent. Thus the effects could not be reproduced by simply changing the currents in the focusing magnets. 


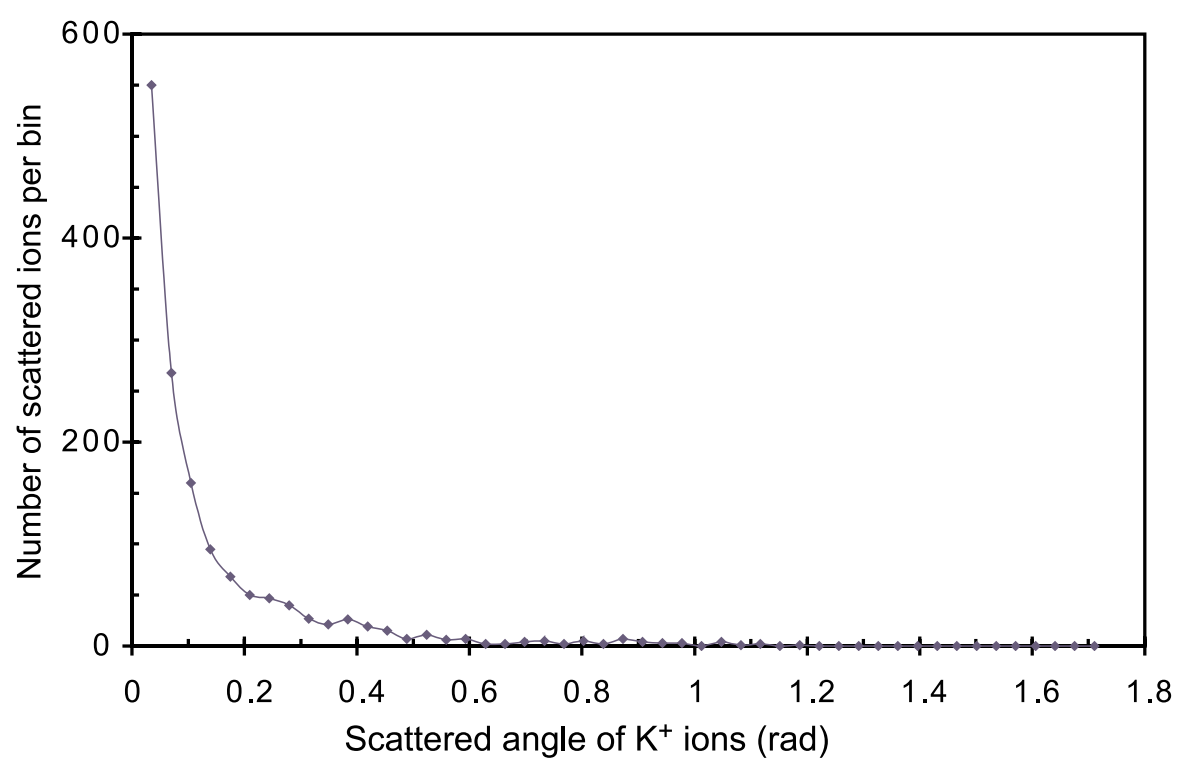

FIG. 7. (Color) Scattered ion distribution versus angle out of plane of incident ion, for two thousand $1.8 \mathrm{MeV} \mathrm{K}^{+}$ions incident on $1 \mu \mathrm{m}$ stainless steel at $89^{\circ}$.

As noted in Sec. II, the predominant source of electrons for long pulses in HIF accelerators is most likely to be ionization of desorbed neutral gas. If the pulse duration is long enough that neutrals released from the walls penetrate deep into the beam interior, then electrons born from ionization of these neutrals will have a density distribution that is sizable where the ion beam density is large. Also, electrons born from ionization of ambient gas will fall within the beam cross section. For constant neutral density, the electron source function would just be proportional to the ion density; the actual electron density would not be, because of the subsequent electron bounce motion in the combined magnetic and potential wells. But certainly the electron density would be concentrated within the beam envelope. Also as noted in Sec. II, for electrons from desorbed neutrals, the electron density will be large within quadrupoles compared to that between quadrupoles if there is acceleration or another loss mechanism in the gaps.

In light of the above considerations our base model electron distribution is chosen to be a constant charge density transverse to the beam direction out to the (elliptical) beam envelope boundary, and axially constant within the nominal axial extent of a quadrupole magnet, but zero outside of these limits. The base case also has the constant charge density the same in each quadrupole. The beam envelope is calculated in the absence of any electron cloud. We run ion beam simulations with this base model, and with a number of perturbations added to this distribution. These are (i) electron density varying from quadrupole to quadrupole (but constant within a quadrupole); (ii) centroid of the electron cloud displaced by an amount which is constant within a quadrupole but varies from quadrupole to quadrupole; (iii) addition of a radially parabolically varying (zero-volume-integrated) density contribution, $\delta n_{e} \sim$ const $\times\left[(x / a)^{2}+(y / b)^{2}-1 / 2\right]$, where $a$ and $b$ are the envelope semiaxes (versus $z$ ), and the constant, which can be positive or negative, is constant within a quadrupole; this perturbation allows the electron cloud to be peaked or hollow; and (iv) addition of extra stretching along one transverse axis and shrinking along the other transverse axis of the electron-cloud envelope, by a factor that is (again) constant in a quadrupole but varying from quadrupole to quadrupole. We consider two types of axial variations: (a) random from quadrupole to quadrupole; and (b) sinusoidally varying from quadrupole to quadrupole [except that we have not done random perturbations of the elliptical distortion, i.e., type (iv)]. In the case of the centroid variation, for the random cases we let the direction of displacement also be random; for sinusoidal cases, the centroid rotates (with fixed fractional displacement relative to the envelope) as one progresses from quadrupole to quadrupole.

Each of the types of perturbations represents a component of variability we can expect in accelerators, as there can be differing amounts and shapes of halo, ion-beam centroid displacements, and shifts in the relative amounts of electrons from gas ionization versus from secondary emission. These variations result from differences in wall conditions, magnet or beam misalignments, and natural oscillations of the beam. The types of random variations are representative of what might be expected from random alignment errors, random changes in wall conditions, and random errors in excitations of magnets. The sinusoidal variations are chosen to explore electrons from, and possible resonance with, breathing, centroid oscillation, and quadrupole oscillation modes of the beam. The sinusoidal cases can be viewed as an extreme 
upper limit on what might be expected from natural variations in beam scrape-off for a properly tuned beam; on the other hand, if a natural mode of the beam is excited, due to some beam detuning (perhaps from the electron cloud itself), then one might expect a resonant, sinusoidally varying component to the electron cloud. The sinusoidal studies should also provide an indication of the ion beam degradation that may be expected to accompany an electron-ion instability which has grown to the specified level of electron perturbation (should such an instability exist in the studied wavelength regime).

All simulations are run for a $2 \mathrm{MeV}$ potassium beam through the same 100-lattice-period quadrupole magnet system described in the preceding section.

For this case of no acceleration, the spatial frequencies for the various beam modes (centroid oscillations, breathing mode, quadrupole oscillations) are easily derived in a continuous-focus approximation; the frequencies for these modes are defined and evaluated, including the effects of the electron space charge, in Appendix B. For the sinusoidal cases we vary the frequency in the vicinity of the nominal resonant frequency to search for a resonance with the beam mode.
In our base case, the electron density in each quadrupole is constant within the beam envelope, and the constant density is the same from quadrupole to quadrupole. The magnets are tuned for a matched beam with no electrons. We show, in Fig. 8, results (an $x-y$ scatter plot, beam current, emittance for various beam fractions, and envelope versus axial position $z$ ) for the base case with an electron density $n_{e}$ that is $20 \%$ of the nominal beam density $n_{b}$ (nominal meaning where the envelope is circular). Even for such a large electron density, there is little beam degradation: a small growth in the emittance, but no observable envelope growth or beam loss. This might have been anticipated; referring to the previously noted rough analogy to modified external focusing, a constant $20 \%$ change in external focusing would have resulted in some beam mismatch, but probably not enough to produce significant emittance or envelope growth.

Figures 9-11 show the results for various types of random perturbations. For all cases, the random component is equal in peak magnitude to the constant component. Hence the electron density for the $20 \%$ mean case varies from 0 to $40 \%$ of the beam density; for axis displacements, the centroid varies from zero to the nomi-
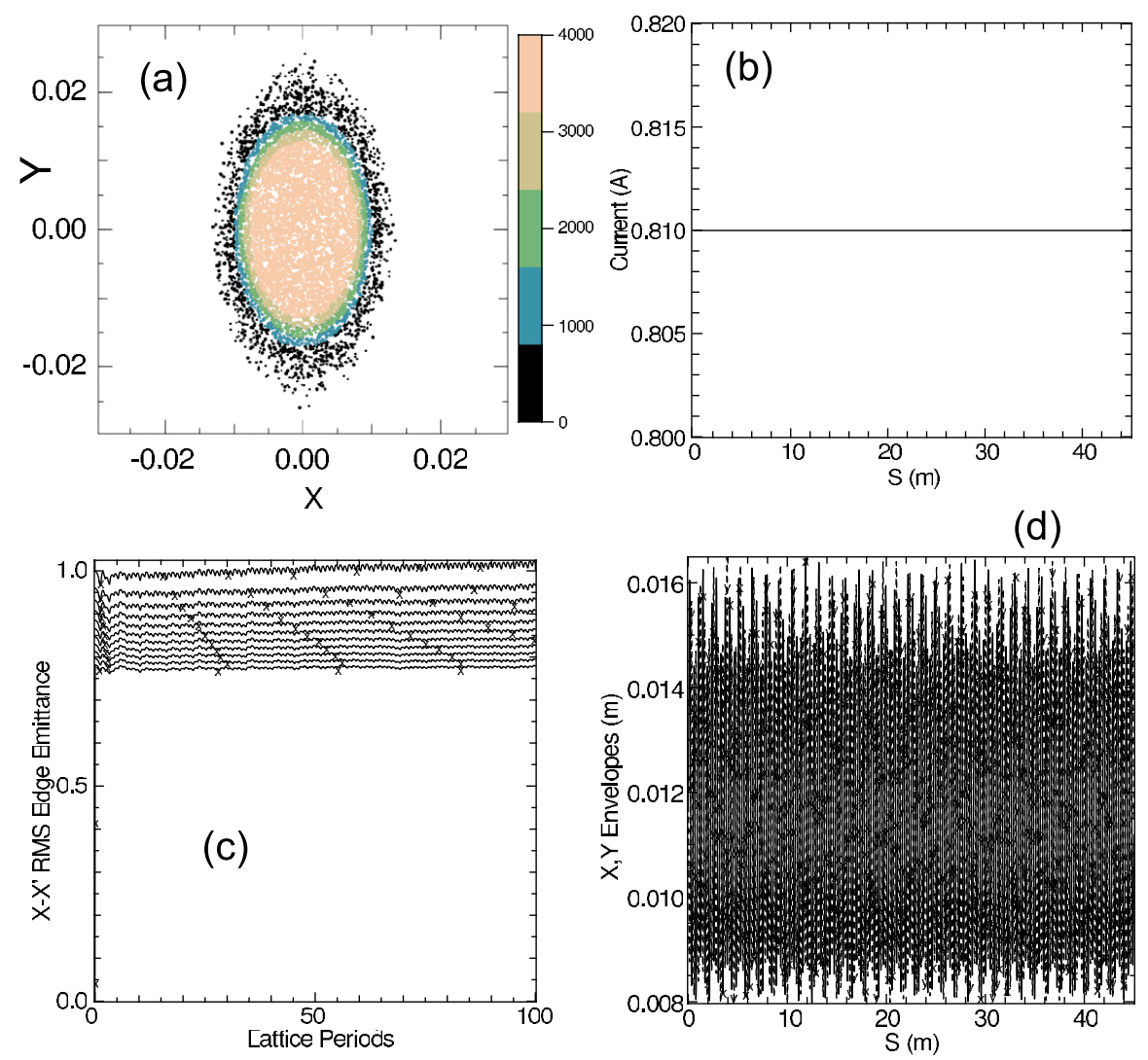

FIG. 8. (Color) Results for fixed $20 \%$ electron density in each magnetic quadrupole: (a) $x$ - $y$ scatter plot of ions near end of magnet array; (b) beam current versus axial position; (c) emittance in $x$ plane versus axial position for various percentages of the beam current enclosed by nested ellipses in phase space (90\% to $100 \%$ in $1 \%$ increments); (d) $x$ and $y$ envelope semiaxes (the rms extents times two) versus axial position. 

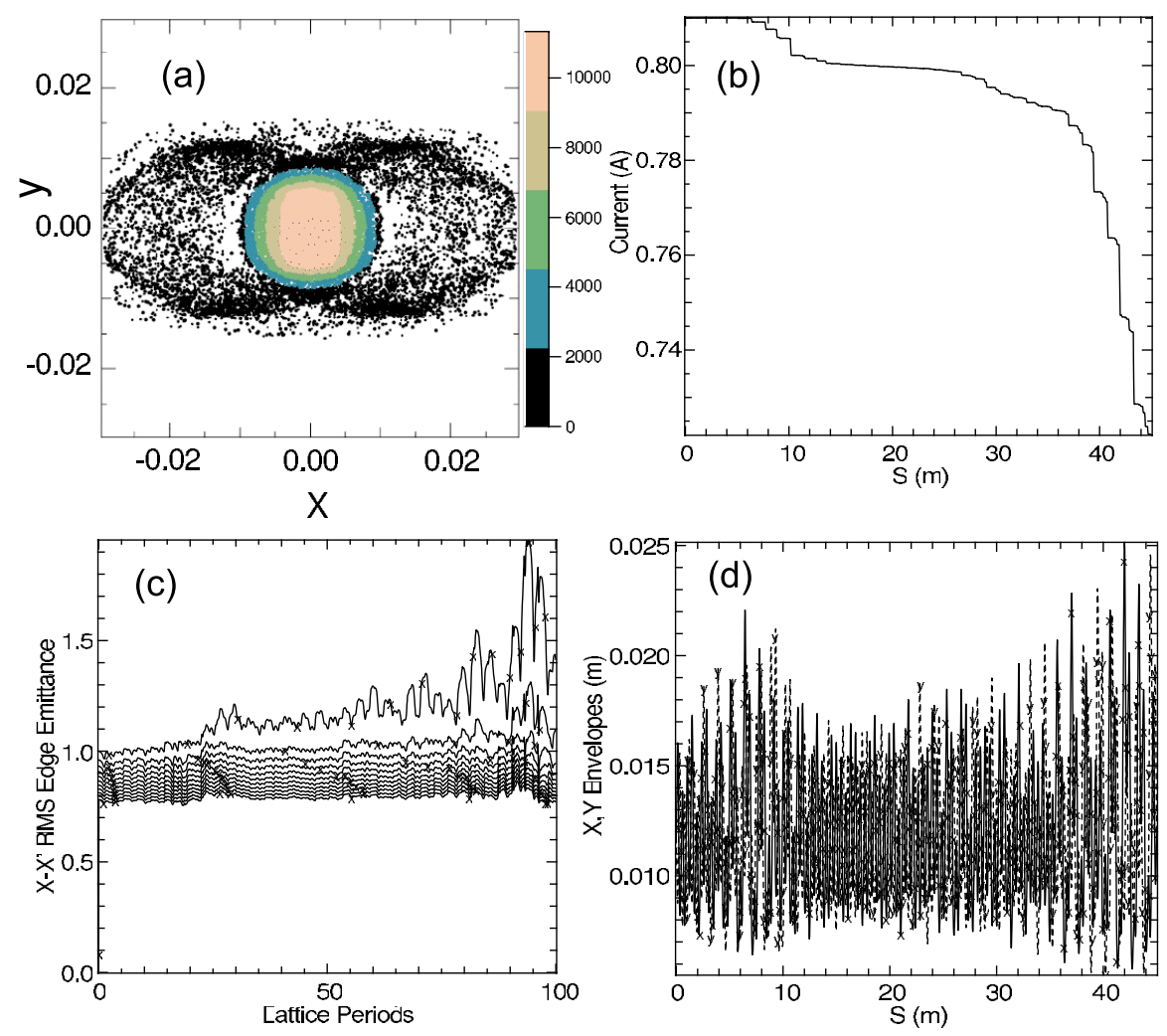

FIG. 9. (Color) Results for random amplitude fluctuations, with $20 \%$ mean electron density and 100\% modulation. (a)-(d) have same significance as in Fig. 8.

nal envelope half-width; for transverse shape variations, the maximum density variation is from zero to double the nominal electron density. These are, obviously, extreme variations. For all cases the shown $x-y$ scatter plot represents a "typical bad slice" chosen from near the end of the simulation. The choice of which plot to show is arbitrary; we pick a case with a relatively large, and in some sense interesting, halo. As is evident, the random amplitude case is the most effective in degrading the beam; a $20 \%$ mean electron density leads to a loss of $10 \%$ of the beam over the 100-quadrupole system. Examination of the set of plots in Fig. 9 indicates that the major incidents of beam loss are associated with periods where the beam envelope is especially large and in fact close to the wall, and also that the emittance grows at about the same $z$ locations. This emittance growth is limited to the outermost class of ions, which experience anharmonic forces outside of the boundary of the model electron cloud. The number of ions experiencing such anharmonic motion is biggest where the envelope has its greatest extent. We expect that an integration of envelope equations with an appropriately modified charge density would yield similar results for the envelope evolution.

Figures 10 and 11 indicate that random offsets and random beam shape variations are considerably less effective than random amplitude variations in degrading beam quality, especially for the outermost ions. One might have supposed that these sorts of perturbations would be particularly effective in coupling to centroid oscillations and breathing modes of the beam, but apparently this is not the case.

Since the worst type of random variability is amplitude variations, it is of interest to determine how beam degradation depends on the size of this variation. Hence we have performed a scan in this parameter. In all cases the mean and fluctuating components are varied together (100\% modulation). We have also done selected cases with different constant components and found little difference. In fact the constant component can be completely eliminated with little change; the variable component is what matters. (This is consistent with the $20 \%$ constant electron-density case producing little effect on the beam.) Figure 12 shows the results of this scan for beam current loss. Roughly, it appears that the beam current loss scales as about $\left(n_{e} / n_{b}\right)^{p}$ with $p$ about 3.54 , but saturates at about $10 \%$ beam current loss for $15 \%$ $n_{e} / n_{b}$. We have not explored higher fractional electron densities. If there is a saturation, it may be related to an observation about the $20 \% n_{e} / n_{b}$ case: there is rapid envelope growth and loss of about a percent of the beam in the first fifth of the magnet system, but thereafter the beam anneals and the subsequent history is very similar to the $15 \%$ case. 

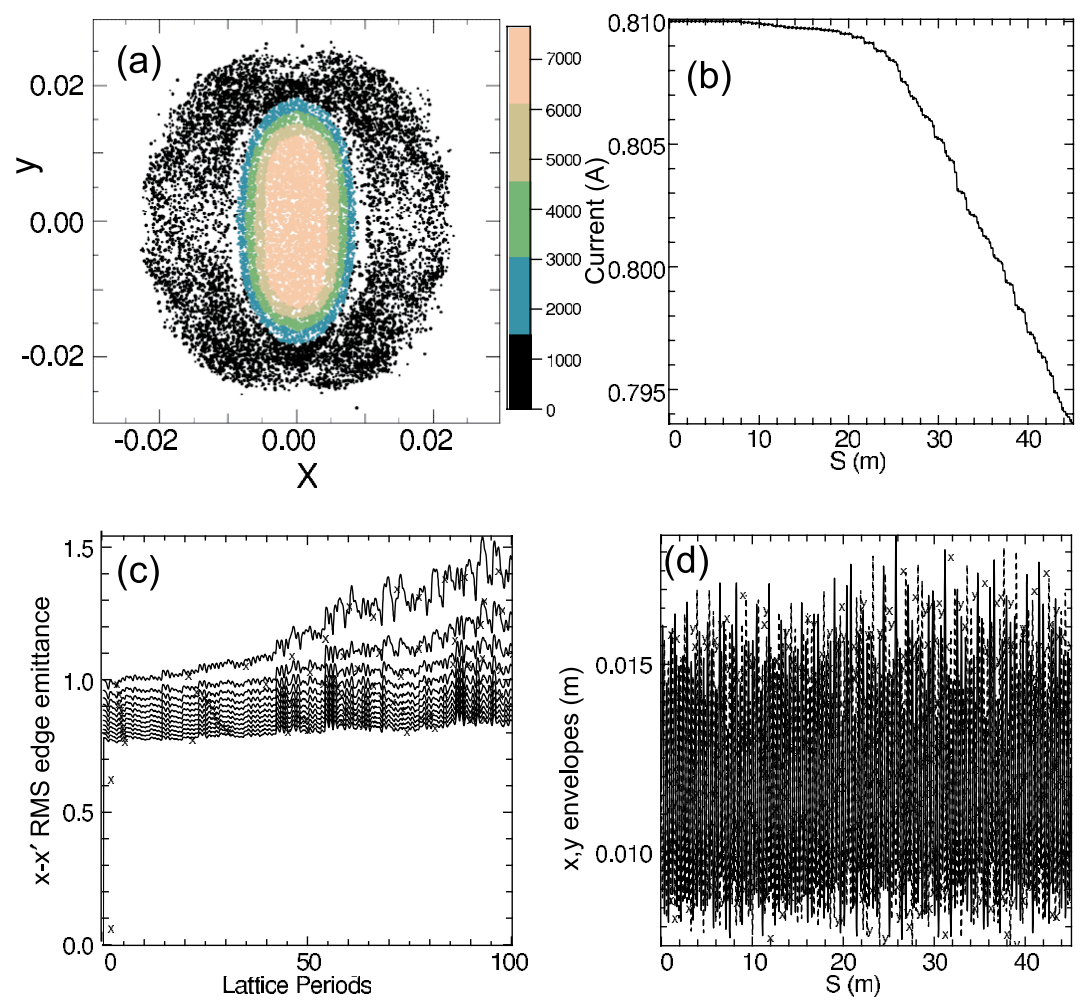

FIG. 10. (Color) Results for random electron-cloud centroid shifts, with $20 \%$ mean electron density and shifts out to $100 \%$ of the envelope radius. (a)-(d) have same significance as in Fig. 8.
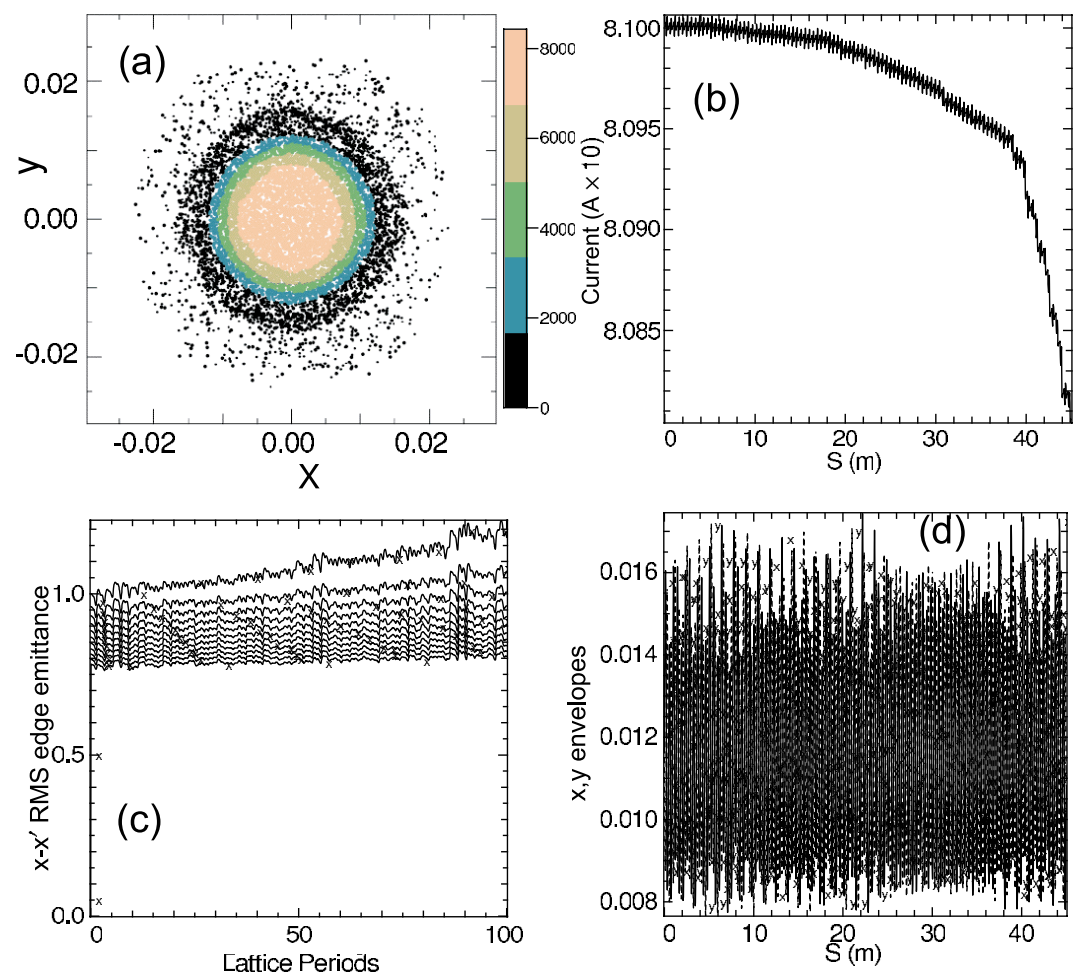

FIG. 11. (Color) Results for random electron-cloud radial shape variations, with $20 \%$ mean electron density and $100 \%$ modulation (radial density variation from as little as zero to as much as twice the mean). (a)-(d) have same significance as in Fig. 8. 


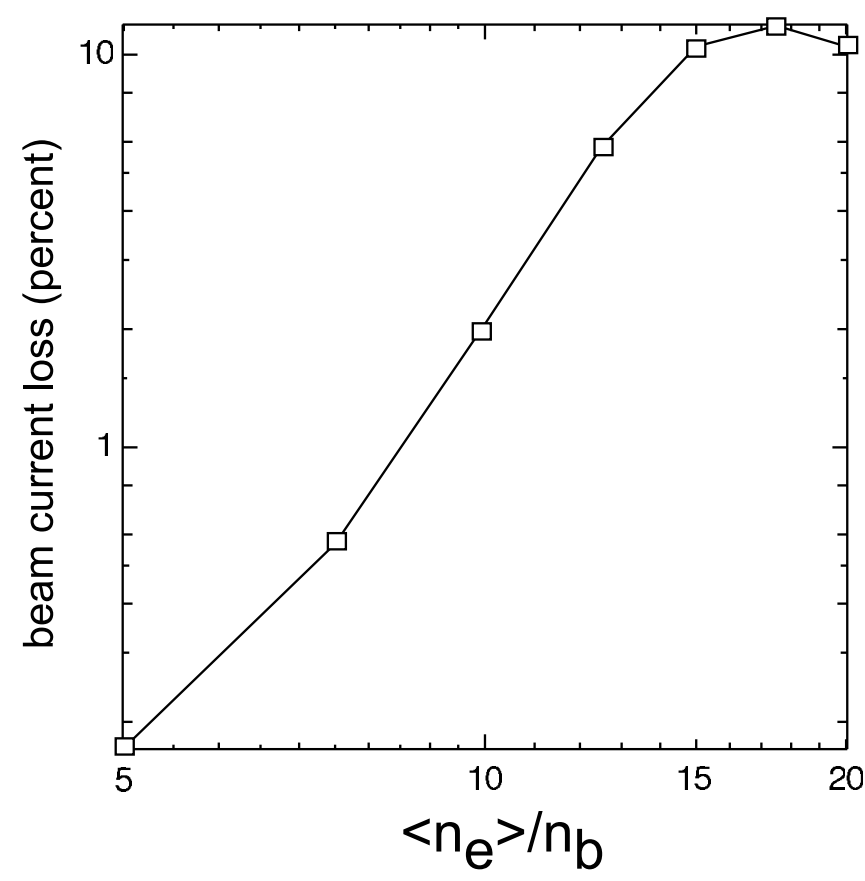

FIG. 12. Fractional beam loss versus rms electron density variation, which in all cases is the same as the mean electron density and in all cases is normalized to the beam density.

Next we turn to the sinusoidally varying cases. Figure 13 shows the fraction of beam loss versus perturbation wavelength, normalized to the lattice period. This is for sinusoidally (versus quadrupole number) varying density, with three values $(2.5 \%, 4 \%, 5 \%$ of the beam density) for the mean electron density. We see that there is

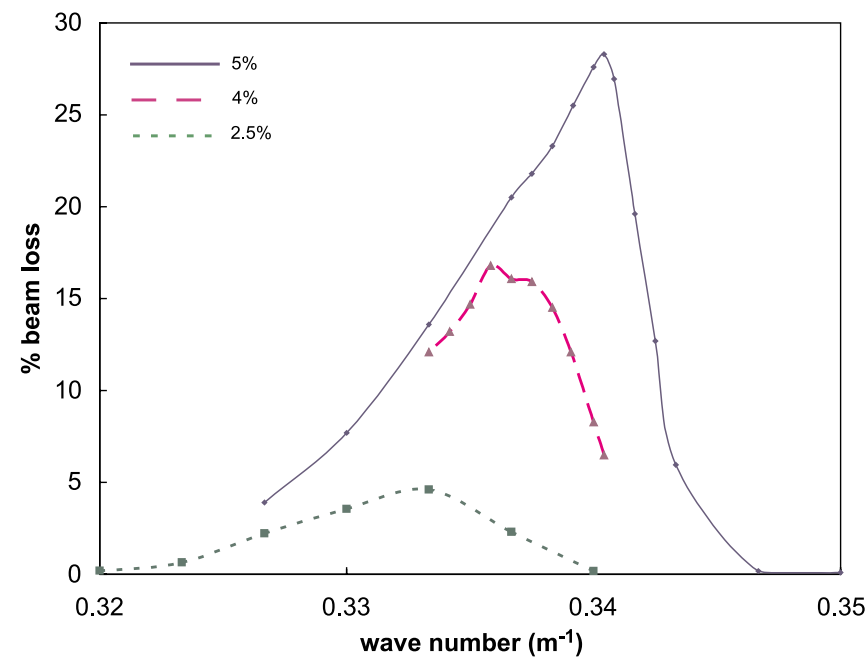

FIG. 13. (Color) Fractional beam loss versus perturbation wave number for electron cloud density that varies sinusoidally with quadrupole number. Mean electron density is 5\% (solid curve), $4 \%$ (dashed curve), or $2.5 \%$ (dotted curve) of central beam density, and modulation is $100 \%$. Wave number is normalized to inverse of lattice period. a sharp maximum, close to the nominal breathing-mode resonance, corresponding, for $5 \%$ mean density, to a beam loss of $28 \%$. We notice that the loss is comparable to the $20 \%$ mean randomly varying amplitude case. From the maxima of the three curves, one can infer that the current loss is roughly scaling as $\left(n_{e} / n_{b}\right)^{p}$, with $p \sim 2.3-2.6$.

Figure 14 shows the $x-y$ scatter, emittance growth, envelope growth, and beam current at the peak of the $5 \%$-density resonance curve of Fig. 13. Note, we have repeated this simulation (at a fixed perturbation wavelength) for several different initial phases of the sinusoidal perturbation. This was done to determine whether the impact on the beam was the result of a coincidence of an existing beam oscillation phase and the perturbation phase, or the result of the electron-cloud perturbation resonantly driving beam envelope oscillations. While the results differ in detail, the macroscopic results - including the net degradation of beam current, emittance, and envelope - were similar, suggesting that the electron cloud resonantly drives the beam envelope oscillations. One can in fact calculate secular spatial growth from linearized beam envelope equations with a driving sinusoidal perturbation in the beam perveance; the spatial growth rate agrees to within about a factor of 2 with the simulation results (the simulation grows more slowly). We will discuss the envelope calculation further in the next section. There are several effects which may contribute to the residual discrepancy: the analytic calculation assumes an electron density which varies sinusoidally in $z$, whereas the model electron distribution in the simulations is constant within a quadrupole magnet, and zero in the gaps between magnets. The analytic calculation models a fractional electron density perturbation by multiplying the perveance by that fraction, which is not correct once the ion envelope shape departs from its electronfree form. And finally, in the kinetic simulation, all characteristic frequencies are spreads about a mean, tending to decrease coherence compared with envelope calculations.

Figures 15 and 16 show the quantities corresponding to Figs. 13 and 14 for a radially varying electron cloud shape (still resonant with breathing modes). In this case the mean electron density is $10 \%$ of the ion beam density. Again there is a sharp maximum versus perturbation frequency. We notice that the maximum beam loss for this case is smaller than that for amplitude variations (even at the larger mean electron density), but is still larger than any of the random variations studied.

Figures 17 and 18 show the corresponding quantities for when the electron-cloud centroid is varied sinusoidally at frequencies in the vicinity of that for centroid oscillations. Again, the electron density in the cloud is $10 \%$. Once again a resonance is found, with a peak beam current loss about a third of that for the radial shape variation reso- 

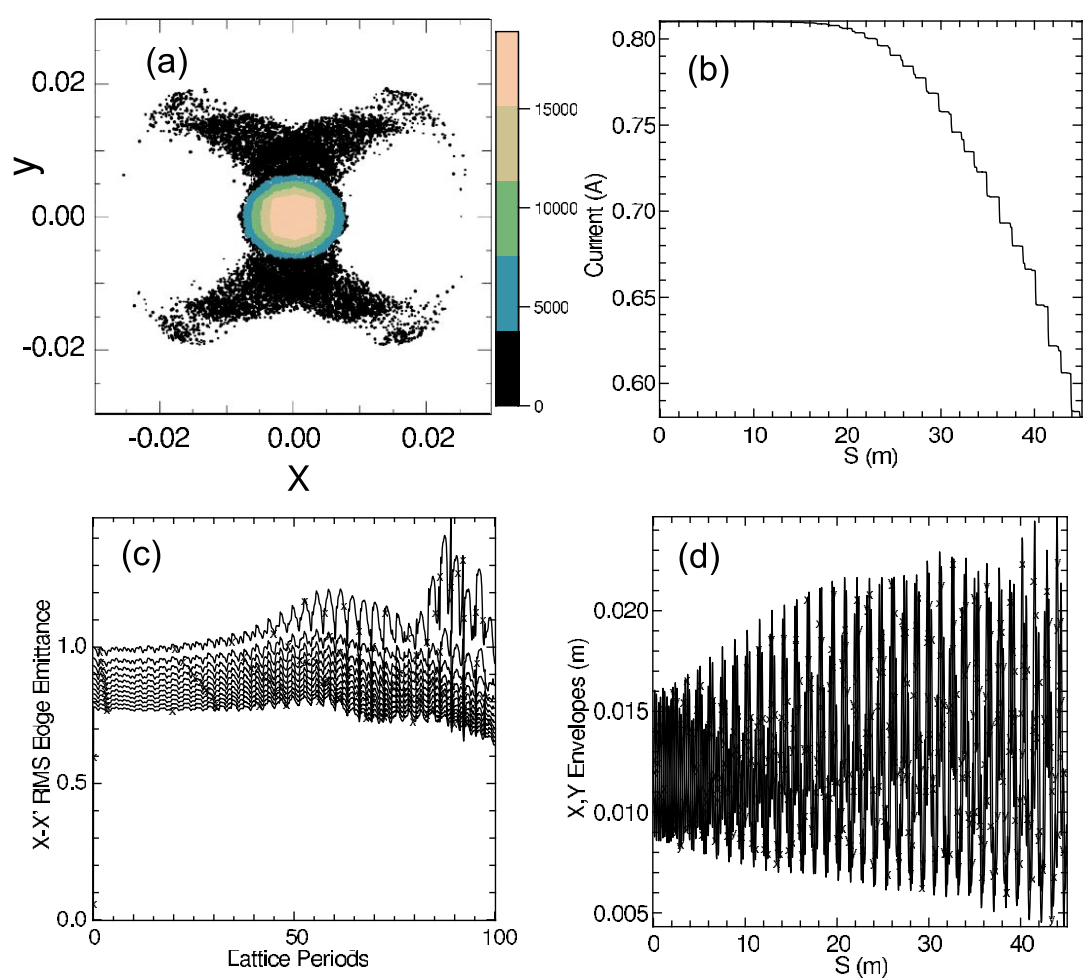

FIG. 14. (Color) Results for sinusoidally varying electron density at the peak of the $5 \%$ curve in Fig. 13. (a)-(d) have same significance as in Fig. 8.

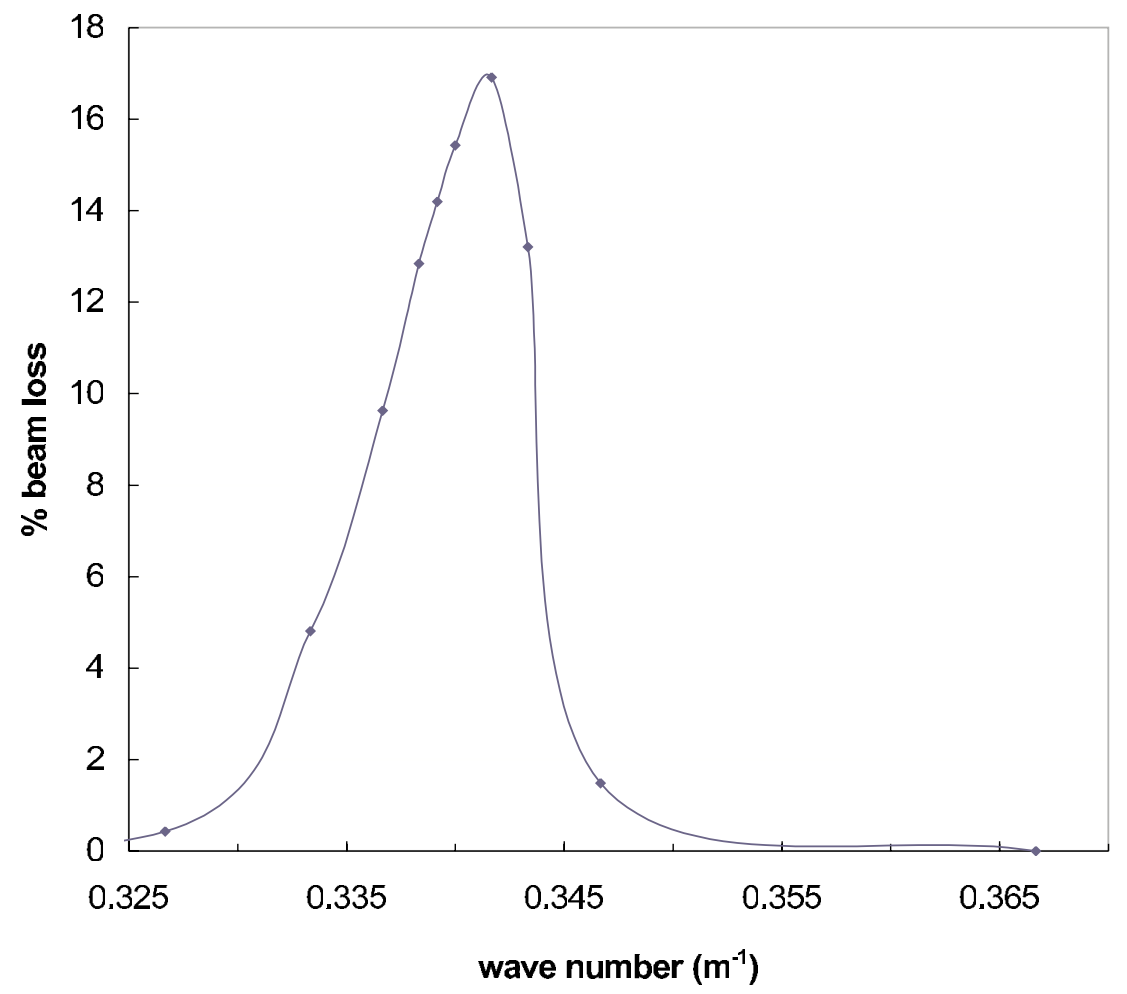

FIG. 15. (Color) Fractional beam loss versus perturbation wave number for electron-cloud radial shape that varies sinusoidally with quadrupole number. Electron density has a mean of $10 \%$ of central beam density and at peak of sine varies from zero to twice the mean. Wave number is normalized to inverse of lattice period. 

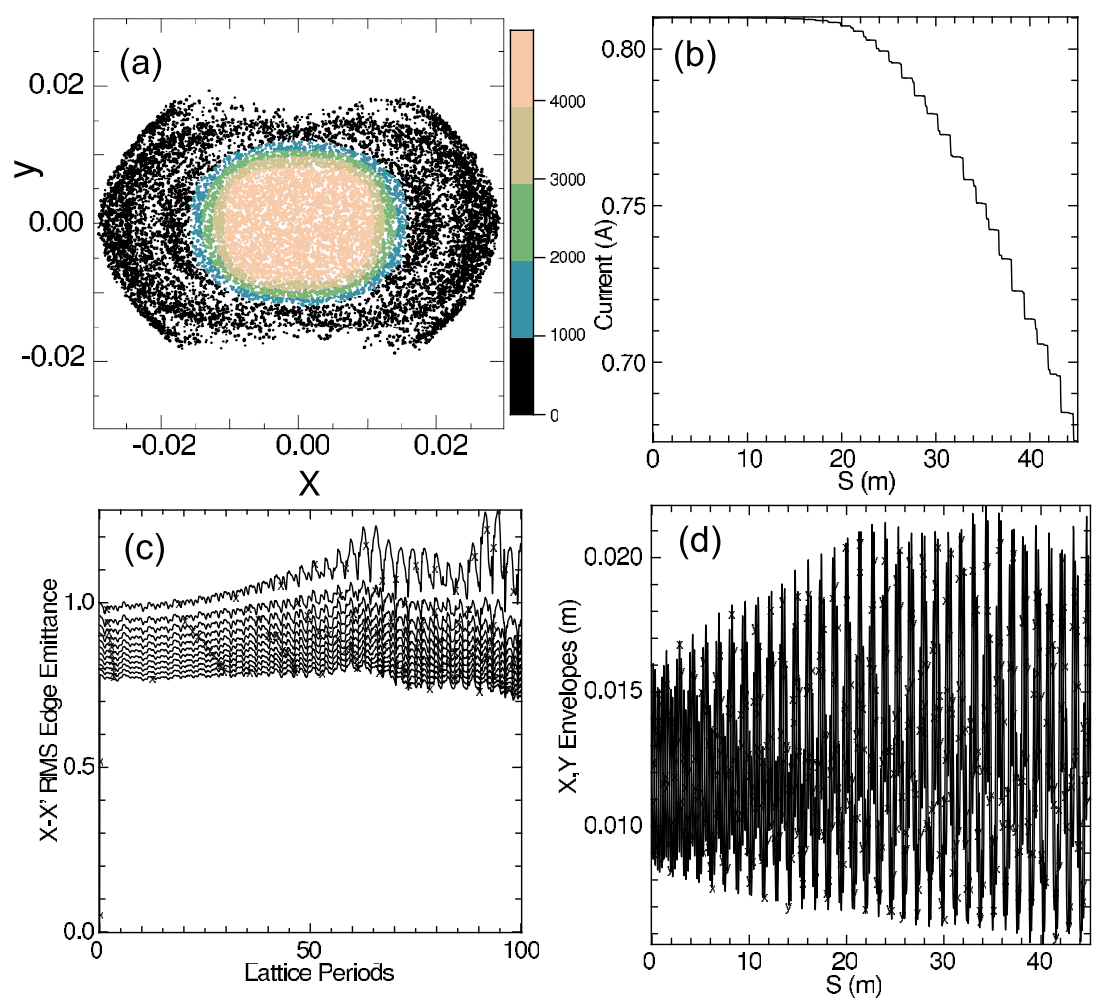

FIG. 16. (Color) Results for sinusoidally varying radial shape of electron density at the peak in Fig. 15. (a)-(d) have same significance as in Fig. 8.

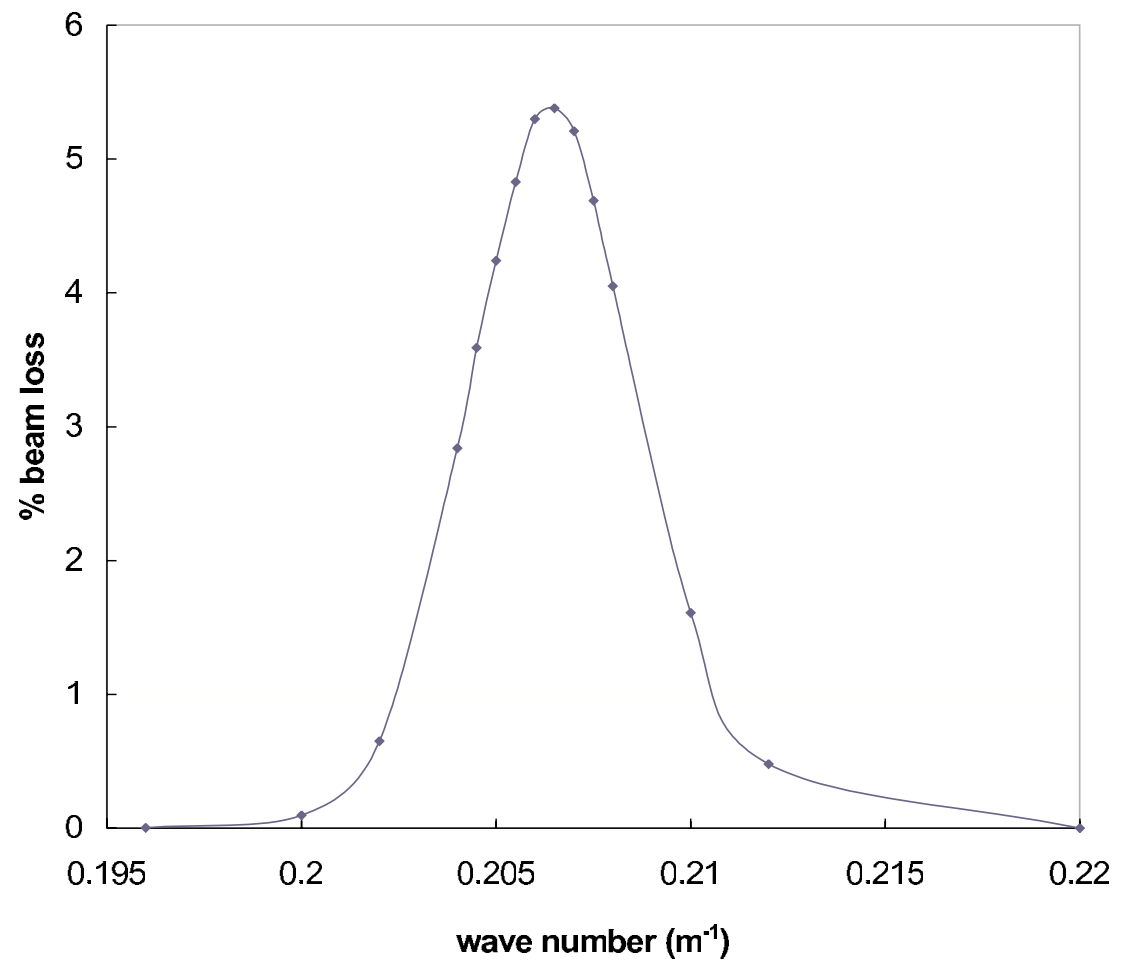

FIG. 17. (Color) Fractional beam loss versus perturbation wave number for electron cloud offset that rotates sinusoidally with quadrupole number. Electron density is $10 \%$ of central beam density and offset is local envelope radius. Wave number is normalized to inverse of lattice period. 

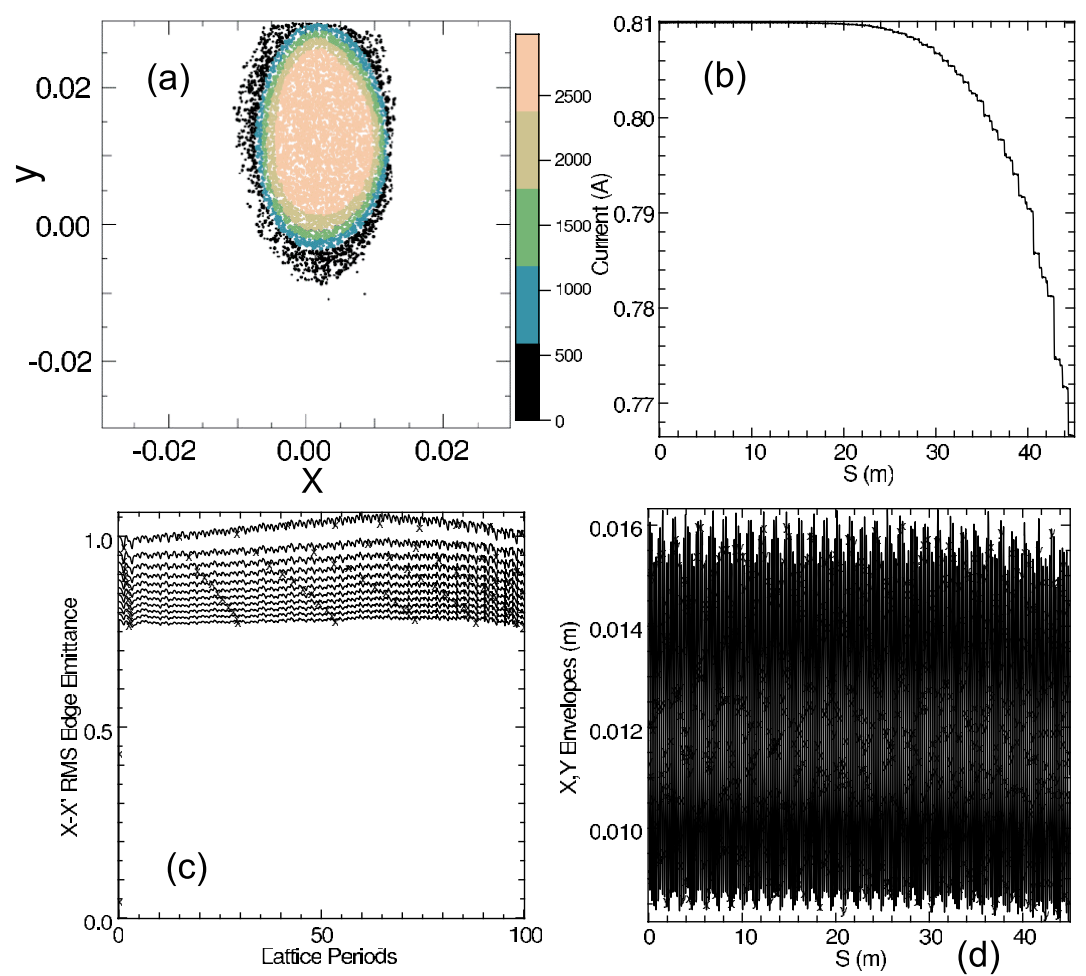

FIG. 18. (Color) Results for sinusoidally varying centroid position at the peak in Fig. 17. (a)-(d) have same significance as in Fig. 8.

nance, and still larger than any of the random variations at comparable electron density.

Figures 19 and 20 show the corresponding quantities for factor of 2 variations in the ellipticity, again for electron density $10 \%$ of the beam density. Here, the resonance is with quadrupolar distortion modes of the ion beam. Again there is a distinct resonance, but the effect on the ion beam, as measured by beam loss, is the weakest of the resonant interactions consideredonly around $4 \%$. On the other hand, the effect on the

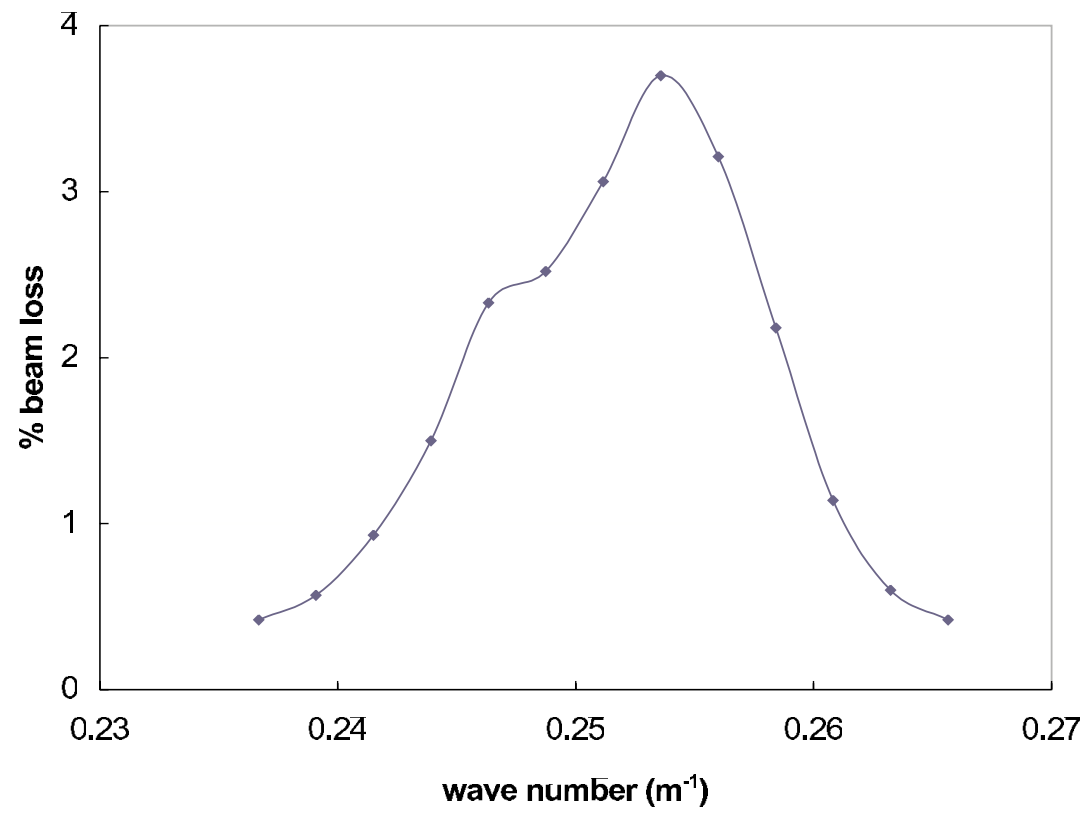

FIG. 19. (Color) Fractional beam loss versus perturbation wave number for electron cloud elliptical distortion that varies sinusoidally with quadrupole number. Electron density is $10 \%$ of central beam density. Maximum enhancement or degradation of ellipticity is a factor of 2 . Wave number is normalized to inverse of lattice period. 

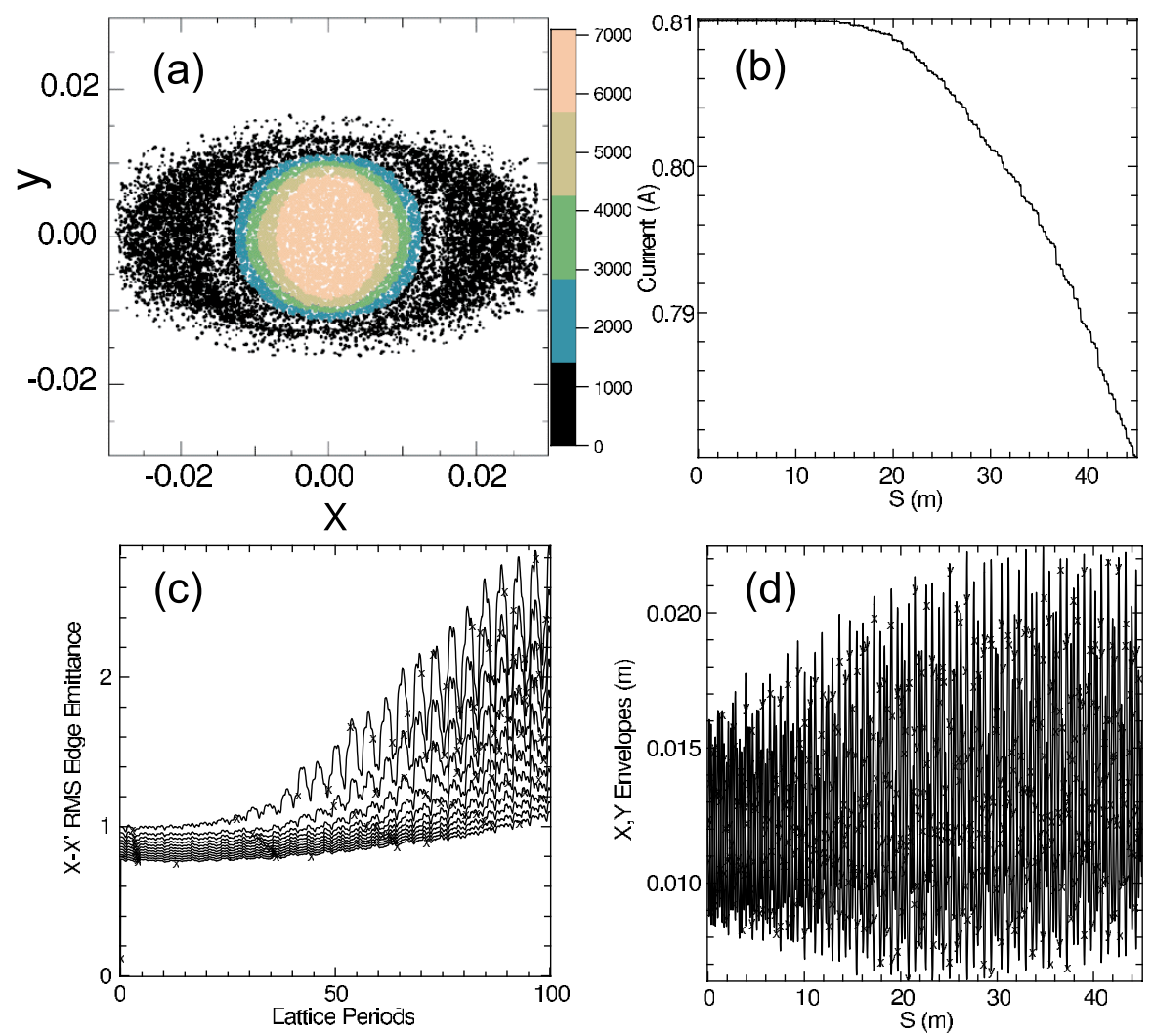

FIG. 20. (Color) Results for sinusoidally varying ellipticity of electron cloud at the peak in Fig. 19. (a)-(d) have same significance as in Fig. 8.

emittance is significant, and it penetrates farther in from the edge of the beam; this is in marked contrast to all of the other perturbations, where emittance growth resulted mostly from the outer few percent of beam particles.

We note that, for sinusoidal amplitude variations, there was no discernible resonance when the frequency was scanned through the range of the centroid and quadrupole resonances. This is in marked contrast to the observations of Figs. 13 and 14. This is expected: the transverse-plane symmetry of the amplitude variation implies that this perturbation cannot couple to centroid oscillations or quadrupole-mode envelope oscillations, but can effectively couple to breathing envelope modes.

\section{DESORPTION INSTABILITY}

The observation that sinusoidal amplitude variations produce especially strong beam current loss suggests the possibility of an instability associated with desorption and subsequent ionization of gas following this resonant beam loss. The scenario is that an electron-cloud variation that is resonant with breathing leads to enhanced desorption and ionization, and since the axial motion of the resultant electrons is limited by the quadrupole magnetic fields, the incremental electron density will have the correct wave number to be resonant with the beam. Given sufficient coincidence of phases, the initial perturbation will grow, giving instability. Because of the stationarity of the electron perturbation, constant wave number is not required for the instability to exist; hence it could occur in an accelerating beam.

To analyze this possibility, we examine linearized equations for the beam envelope following Refs. [11,12]. We restrict attention here to a coasting beam. In the smooth focusing approximation, the mean values of the beam envelope functions $X(z)$ and $Y(z)$ follow the equations

$$
\begin{aligned}
& X^{\prime \prime}+k_{0}^{2} X-\frac{2 Q}{X+Y}-\frac{\varepsilon^{2}}{X^{3}}=0, \\
& Y^{\prime \prime}+k_{0}^{2} Y-\frac{2 Q}{X+Y}-\frac{\varepsilon^{2}}{Y^{3}}=0,
\end{aligned}
$$

where $Q$ is the beam perveance, $\varepsilon$ its emittance, and $k_{0}$ is the wave number for a single particle oscillating in the lattice and is related to the strength and geometry of the lattice quadrupoles. $k_{0}$ is also the natural wave number of oscillations for the beam centroid. The perveance $Q$ is defined as

$$
Q=\frac{q I}{2 \pi \varepsilon_{0} m v_{z}^{3}},
$$

where $q$ and $m$ are, respectively, the charge and mass of 
the beam particles, $v_{z}$ is the average axial beam velocity, $I$ is the beam current, and $\varepsilon_{0}$ is the permittivity of vacuum.

We linearize these equations for breathing-mode (axisymmetric) perturbations, $X=Y=\bar{R}+\tilde{x}$, where $\tilde{x}$ is the envelope amplitude perturbation, to obtain

$$
\tilde{x}^{\prime \prime}+k_{b}^{2} x=-\frac{Q}{n_{b} \bar{R}} \tilde{n}_{e},
$$

where $k_{b}$ is the wavelength for breathing oscillations (defined in Appendix B). In deriving this equation we have made use of the fact that the perveance is linearly proportional to the beam current and hence to the total charge density, so that the perveance perturbation is just $-Q \tilde{n}_{e} / n_{b}$. Also, in this equation the prime $\left(^{\prime}\right)$ means differentiation with respect to the axial coordinate $z$ for a particular beam slice, i.e., for a fixed $z-v_{b} t$.

We note at this point that one can use Eq. (4) to derive the response of the envelope to a specified electron density perturbation, such as used in the studies in the preceding section. We did this for a resonant sinusoidal density perturbation; this led to an estimate of the envelope growth that was about twice as big as the simulation result shown in Fig. 14, as reported in the preceding section.

We proceed with our instability analysis by relating $\tilde{n}_{e}$ to the envelope growth. Specifically, we take

$$
\frac{\partial \tilde{n}_{e}}{\partial t}=n_{b} \tilde{n}_{0} \sigma_{i} v_{b}-\tilde{n}_{e} / \tau_{e},
$$

where $\tilde{n}_{0}$ is the neutral density perturbation (averaged over the beam), and $\tau_{e}$ is the electron perturbation loss time. Following the discussion in Sec. II, this time would be of order of the time to drift through a quadrupole $\tau_{d}$ for the case of acceleration in the gaps, and is of order $N_{b}^{2} \tau_{d}$ for the case of no acceleration, where $N_{b}$ is the number of quadrupoles in half of a breathing-mode wavelength.

The average neutral density perturbation should be related to the neutral flux from the wall in a fashion that builds in some delay from the neutral time of flight. We model this by the relation as

$$
\frac{\partial \tilde{n}_{0}}{\partial t}=\frac{\tilde{n}_{0 \infty}-\tilde{n}_{0}}{\tau_{n}},
$$

where $\tau_{n}$ is an average time of flight for neutrals from the wall to the beam interior, and $\tilde{n}_{0 \infty}$ is the neutral density perturbation that would be present neglecting the time of flight,

$$
\frac{\partial \tilde{n}_{0 \infty}}{\partial t}=\frac{2 \pi r_{w}}{\pi r_{w}^{2}} \kappa_{n} \tilde{\Gamma}_{w} \approx \frac{2}{r_{w}} \kappa_{n} n_{b} \frac{\partial \tilde{x}}{\partial t} .
$$

Here $r_{w}$ is the wall radius, $\kappa_{n}$ is the neutral emission per lost ion, and $\tilde{\Gamma}_{w}$ is the perturbed beam flux to the wall. In the last expression on the right, we have approximated $\tilde{\Gamma}_{w}$ by the beam density times the envelope velocity, which is probably an upper limit (it effectively assumes that in the absence of a perturbation the beam envelope is at the wall).

Finally, we note the connection between $z$ derivatives at constant beam slice (at constant $\zeta \equiv z-v_{b} t$ ) and at constant time $t$, namely,

$$
\left.\frac{\partial}{\partial z}\right|_{\zeta}=\left.\frac{\partial}{\partial z}\right|_{t}+\left.\frac{1}{v_{b}} \frac{\partial}{\partial t}\right|_{z}
$$

Combining Eqs. (4)-(8), and assuming that all linear quantities vary as $\exp (i k z-i \omega t)$, we obtain the dispersion relation

$$
\left(1-i \alpha_{1} \hat{\omega}\right)\left(\hat{\omega}+i \alpha_{2}\right)\left[\hat{\omega}^{2}-2 \hat{\omega}+\left(1-\frac{k_{b}^{2}}{k^{2}}\right)\right]-i \alpha_{3}=0,
$$

where

$$
\alpha_{1}=k v_{b} \tau_{n}, \quad \alpha_{2}=\frac{1}{k v_{b} \tau_{e}}, \quad \alpha_{3}=\frac{2 Q \sigma_{i} \kappa_{n} n_{b}}{\bar{R} r_{w} k^{3}},
$$

and $\hat{\omega}=\omega / k v_{b}$.

Here, we shall assume that $k$ is real and estimate the maximum growth rate. A more accurate procedure would entail a search for the maximum temporal growth rate allowing for convective (spatial) growth as well, and may be expected to yield a somewhat higher growth rate. But, given the degree of approximation already made in treating only transverse averages, in the time-of-flight model, and in the loss flux expression, the real- $k$ approximation should be good enough for our estimation purposes.

One can of course numerically solve Eq. (9) for the four roots for particular values of $\alpha_{1}, \alpha_{2}$, and $\alpha_{3}$, and find the root with the largest $\operatorname{Im} \omega$. But we can obtain scalings by assuming that various constants are large or small. For parameters similar to those of the preceding section, for the fastest-growing root, the appropriate approximations are $\alpha_{3}$ and $\alpha_{2} / \hat{\omega}$ small and $\alpha_{1} \hat{\omega}$ large, in which case the maximum growth rate is at $k=k_{b}$ and is obtained from the imaginary part of $\omega \sim\left(\alpha_{3} / 2 \alpha_{1}\right)^{1 / 3}$, or

$$
\gamma \equiv \operatorname{Im} \hat{\omega} k v_{b}=\frac{1}{2}\left(\frac{\alpha_{3}}{2 \alpha_{1}}\right)^{1 / 3} .
$$

For the parameters of the simulations of the preceding section, we have $\gamma^{-1} \sim 4 \mu \mathrm{s}$, which is about the same as the pulse duration. This value is obtained for perveance $K=0.021, \quad \sigma_{i} \approx 10^{-15} \mathrm{~cm}^{-2}, \quad \kappa_{n} \approx 10^{4}, \quad n_{b}=$ $3.71 \times 10^{9} \mathrm{~cm}^{-3}, \bar{R} \approx 1.12 \mathrm{~cm}, r_{w}=2.9 \mathrm{~cm}$. Note for these parameters our "large" and "small" parameters are not very large and small, $\alpha_{1} \omega_{n} \sim 2.3 \tau_{n}(\mu \mathrm{s}), \alpha_{3} \sim$ 0.26 , and $\alpha_{2} / \omega_{n} \approx 0.15$ for $\tau_{e} \sim 3 \mu \mathrm{s}$.

We expect that this estimate is overly pessimistic. The approximation that the perturbed ion beam flux to the 
wall is the beam density times the perturbed envelope velocity should be an upper limit, and growth would also likely be limited by non-ideal-envelope effects (as was noted in the comparison of the simulations with the envelope predictions for the legislated sinusoidally varying density) and by velocity tilt (the tail of the beam having a different velocity from the head). Hence our conclusion is that an instability exists but is quite mild.

\section{BRIDGING ELECTRON AND ION TIME SCALES}

A quantitative description of electrons in HIF accelerators requires following them through both strongly magnetized and unmagnetized regions. By "strongly," we mean that the electron-cyclotron period and gyroradius are short compared to other electron time and length scales. Such simulation presents a challenge, particularly if the simulation is to also follow the longer time scales of the ion dynamics. Full-orbit electron dynamics is costly as one must take time steps smaller than the electron-cyclotron period within the magnetic quadrupoles. Drift kinetics or gyrokinetics resolves this problem (one averages over the fast gyro motion to describe particle motions in terms of a fast parallel flow and a small drift across magnetic field lines), but only in the magnets and away from the field nulls; the approximations break down near the nulls and in the gaps between magnets.
We have devised a scheme that entails interpolating between full ion dynamics (as implemented via a Boris mover [13]) and drift kinetics [14]. With the interpolation scheme, one is allowed to take time steps that are large compared to the electron-cyclotron period, and still obtain physically correct parallel dynamics, cross-field drift, and gyroradius (at least in the field and potential configurations where we have checked). What is sacrificed is the frequency of gyration, which is reduced from the actual gyrofrequency. Hence, so long as one is not dealing with phenomena operating on the scale of the highest gyrofrequencies in the system, one can take time steps without regard to the gyrofrequency, so long as the steps resolve the phenomena of interest. This scheme will be described in detail in a separate paper (in preparation).

Here, we show a sample application to HIF, specifically the electron-cloud calculation of Sec. III. In this case, the time step for the interpolation scheme is chosen to resolve the electron bounce motion, which is comparable to the time scale needed to resolve an electron-ion two-stream instability [15], or to resolve ions passing through the quadrupole fringe fields in the high-energy end of a driver. Figure 21 shows the results of the same sequence of calculations as in Sec. III, except that the interpolated mover is used to push the electrons and the time step is 25 times larger (and the electron code run nearly that much shorter). Comparing Figs. 5 and 21, we see that the interpolated and small-time-step movers produce almost identical results (note that the color scales are identical).

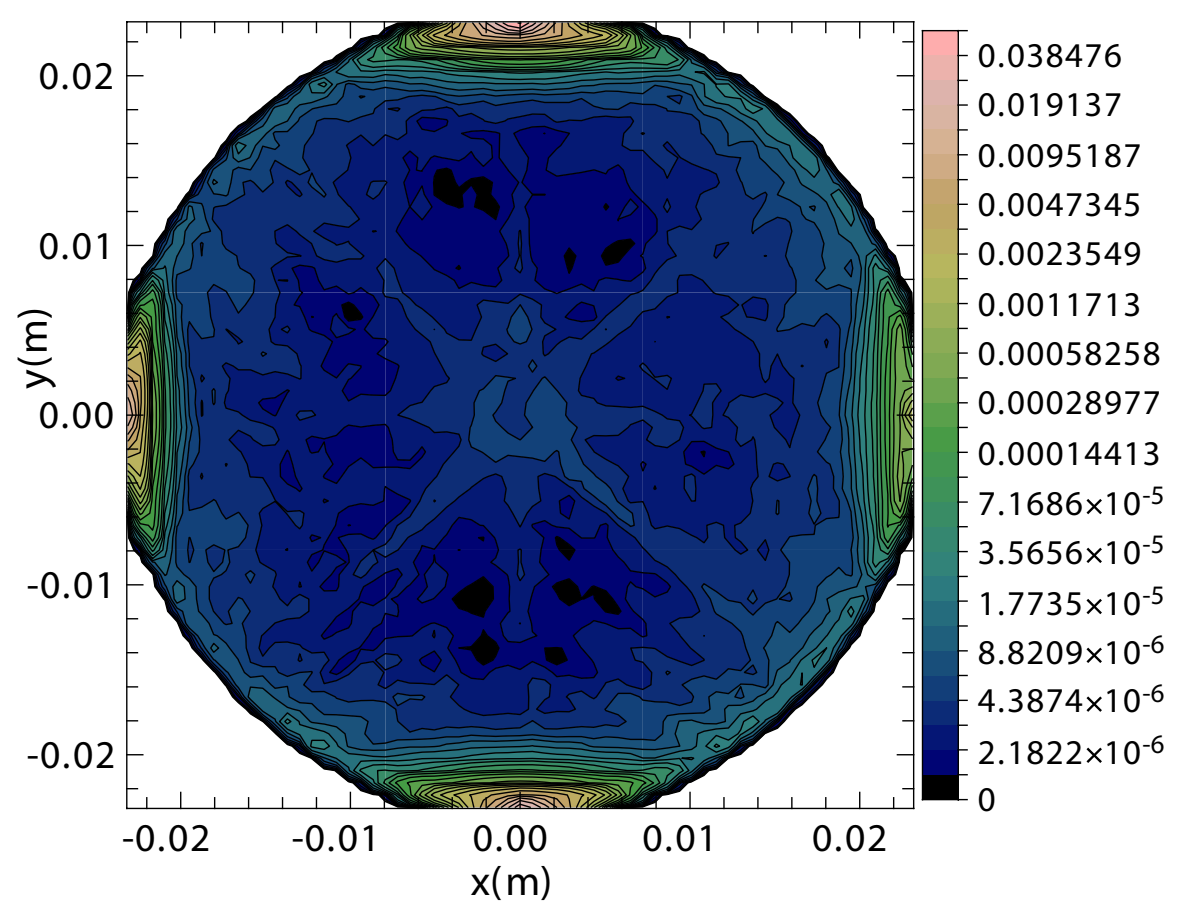

FIG. 21. (Color) $x-y$ projection of electron cloud density retaining electrons desorbed by impact of primary beam and scattered ions at walls, calculated using interpolated electron mover. 


\section{SUMMARY AND DISCUSSION}

Induction accelerators for heavy-ion fusion possess attributes that significantly impact electron-cloud physics. In particular, the combination of high current, large fill factor, long pulse length, and difficulty of wall conditioning imply that the dominant electron source is ionization of neutrals desorbed from walls in current experiments with multi- $\mu$ s pulses, and in the low-energy end of a driver. For shorter pulse experiments, and at the high-energy end of a driver, electrons produced at or near walls, from electron desorption or from ionization of neutral gas near walls, will dominate. The precise balance depends on the composition and energy distribution of the desorbed neutrals and the pulse length. The same combination of factors listed above implies that self-consistent, simultaneous modeling of electron and ion dynamics is required for a quantitative assessment of electron-cloud buildup. We have implemented and discussed here elements of such a modeling capability.

Our simulation of the dynamics of electrons from direct electron desorption (due to impact of ions) indicates the importance of including ion scattering at walls. Whereas the primary beam ions are lost exclusively at pipe locations where the magnetic field lines confine electrons to a region near their birth positions, scattered ions can reach field wall positions intercepted by field lines that extend deep into the beam interior.

We have undertaken a series of simulations with prescribed (model) electron clouds, which mock up distributions that one might expect from ionization of desorbed neutrals in long pulse accelerators (or neutrals from ambient gas). These simulations are useful for assessing the tolerable level of electron density, and also provide insight into possible instabilities, such as the one reported in Sec. V. The results presented here indicate that electrons are most detrimental to ion beam quality when the amplitude varies from quadrupole to quadrupole, and a density variation that is resonant with beam breathing modes is considerably more effective than a random variation in producing beam loss and degradation of beam quality. For our model problem with a 100 latticeperiod system, a mean $5 \%$ electron population, with a $100 \%$ resonant modulation, leads to loss of $28 \%$ of the beam. For random variations, even a $10 \%$ mean electron density, still with $100 \%$ modulation, leads to loss of only about $2 \%$ of the beam; the $20 \%$ case shown in Fig. 9 results in a $10 \%$ beam loss. Random variations in electron-cloud shape or centroid are considerably less effective in degrading beam quality. Resonant variations in electron-cloud shape (resonating with the breathing mode) or in centroid position (resonating with centroid oscillations) are detrimental to beam quality, but for the perturbations tested not as detrimental as resonant amplitude variations. We note that we have considered only a limited class of possible resonant perturbations; one might imagine choosing frequencies and perturbation forms that would resonate with higher-order internal space-charge modes. However, we expect that the coupling should be strongest to the lowest-order bulk modes of the beam, i.e., the ones considered.

It is evident from examination of the $x-y$ scatter plots, even the few shown, that the nature of the beam degradation is more complex than is captured in the line plots of emittance, envelope, and beam current. In particular, we see from Fig. 9(a) a beam with a nearly circular core but a strongly elliptical (and in fact reaching the wall) halo. The same beam, further along, has a core that is elliptical but has a nearly circular halo (Fig. 22). The elliptical distortion of the main beam would suggest ion scrape-off only on field lines that are nearly tangent to the wall. However, from this latter plot we see that the halo resulting from the electron cloud can be much more symmetric, implying scrape-off on field lines that penetrate deep into the beam interior. This would lead to a relatively larger role for secondary electrons than one would otherwise infer. From the sequence of the two scatter plots, we infer that, not unexpectedly, the ions in the halo are experiencing different oscillation frequencies from those in the core. In Fig. 14(a), a different phenomenon is occurring, leading to halo that is actually concentrated (at this instant) near the quadrupole field lines that penetrate deep into the plasma interior.

There are several overall conclusions to be drawn from this study: (1) Heavy-ion beams are actually surprisingly robust to electron clouds. A priori there was some expectation that even a few tenths of a percent electrons would be devastating. Even for resonant sinusoidally varying electron clouds with $100 \%$ modulation, electron densities

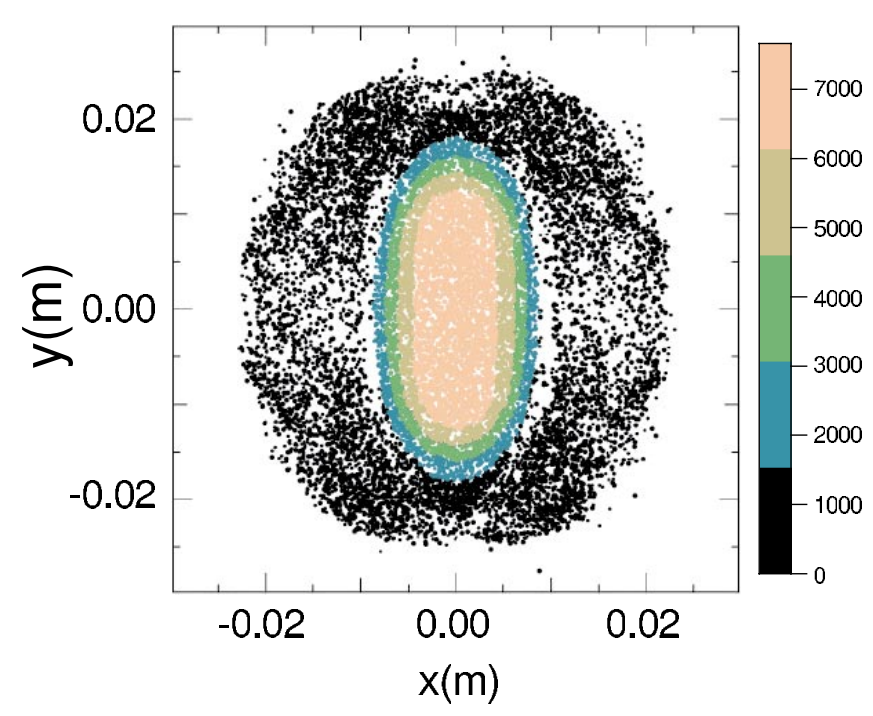

FIG. 22. (Color) $x-y$ scatter plot for random electron density perturbation; same as Fig. 9(a) but a later slice. 
in the range of several percent of the beam density are required for substantial beam degradation of a 200quadrupole system. A constant (from quadrupole to quadrupole) electron density, such as might be caused by ionization of the base pressure of background gas, results in negligible beam loss and envelope growth, and only very minor emittance growth. (2) Electron cloud density modulation resonant with the beam breathing mode is the most dangerous kind of perturbation (at least based on beam loss). (3) Elliptical distortions which resonate with beam quadrupole modes have a global effect on beam emittance (related to the effect of a beam mismatch), even though the beam current loss they produce is relatively modest. (4) Electron-cloud impact on ion beam propagation is a rich and complex subject, and is not well characterized by the evolution of a few low-order beam moments.

The strong effect of density perturbations resonant with breathing-mode oscillations suggests the possibility of a desorption-driven instability. A simple estimate of the growth rate based on linearized envelope equations suggests a moderate growth rate, comparable to the beam pulse duration for HCX-like parameters. Effects not included in the analysis, such as beam velocity tilt and nonideal-envelope phenomena (e.g., halo particles having different resonant frequencies than those in the core), should further limit growth.

Finally, we briefly described a scheme for efficiently simulating electrons which move over a wide range of magnetic-field strengths, by interpolating between a drift-kinetic and full-orbit particle push. We also present a sample electron-cloud calculation from this scheme, which agrees well with one obtained from a full electron dynamics calculation with a 25 times smaller time step. This scheme enhances the prospects for ion beam simulation with self-consistent electron and ion dynamics.

\section{ACKNOWLEDGMENTS}

We thank D. Grote for invaluable assistance with the WARP code. This work was performed under the auspices of the U.S. Department of Energy by University of California Lawrence Livermore National Laboratory under Contract No. W7405-ENG-48 and Lawrence Berkeley National Laboratory under Contract No. DEAC03-76F00098, and under an SBIR grant at TechX.

\section{APPENDIX A: BEAM, LATTICE, AND SIMULATION PARAMETERS}

For concreteness, a particular beam was chosen as the basis of the simulations. The simulated beam is representative of the beam in the HCX apparatus at LBNL. The key parameters describing the beam, the accelerator system, and the simulation are given in Table I.
TABLE I. Beam, lattice, and simulation parameters.

\begin{tabular}{lc}
\hline \hline \multicolumn{1}{c}{ Parameter } & Value \\
\hline Mass & $38.9637 \mathrm{amu}$ (potassium) \\
Charge & +1 \\
Initial current & $810 \mathrm{~mA}$ \\
Initial emittance (unnormalized) & $100 \mathrm{~mm} \mathrm{mrad}$ \\
Initial perveance & $1.1565 \times 10^{-3}$ \\
Energy & $2 \mathrm{MeV}$ \\
Initial dimensions & $=b_{0}=1.17344 \mathrm{~cm}$ \\
Initial divergences & $a_{0}^{\prime}=-b_{0}^{\prime}=-34.616 \mathrm{mrad}$ \\
Initial distribution & Semi-Gaussian \\
Undepressed phase advance & $80^{\circ}$ \\
Depressed phase advance & $19.8^{\circ}$ \\
Lattice period & $45 \mathrm{~cm}$ \\
Number of lattice periods & 100 \\
Quadrupole lattice occupancy & 0.453 \\
Quadrupole syncopation factor & 0.248 \\
Field gradient & $90.51 \mathrm{~T} / \mathrm{m}$ \\
Pipe radius & $2.948 \mathrm{~cm}$ \\
Number of simulation grid cells & $256 \times 256$ \\
Number of macroparticles & 400000 \\
\hline
\end{tabular}

\section{APPENDIX B: WAVE NUMBERS OF THE NATURAL MODES OF OSCILLATIONS}

We summarize here the wave numbers of the natural modes of oscillations for charged particle beams in a FODO lattice. These are slow variations of the beam envelope superposed on the alternating elliptical distortion of the beam envelope that occurs every lattice period due to the action of the alternating-polarity quadrupole magnets.

The natural modes are breathing (where the principal axes of the beam envelope cross section oscillate in phase), quadrupole oscillations (where the principal axes oscillate $180^{\circ}$ out of phase), and centroid oscillations. The oscillation frequencies can be derived from analysis of the smooth-focus envelope equations (1) and (2) following Refs. [11,12]. We account for the presence of electrons through their partial neutralization of the beam space charge, which is in turn modeled by reducing the perveance $Q$ (defined in Sec. V) by the (assumed constant) factor $1-f$, where $f=n_{e} / n_{i}$. We give here the results for the normal mode wavelengths. They are

$$
k_{b}^{*}=\sqrt{2 k_{0}^{* 2}+2 k^{* 2}},
$$

for the breathing mode, and

$$
k_{q}^{*}=\sqrt{k_{0}^{* 2}+3 k^{* 2}},
$$

for the quadrupole mode, with

$$
k_{0}^{*}=\sqrt{k_{0}^{2}+\frac{S^{2} f Q}{\bar{R}^{2}},}
$$


TABLE II. Scaled wave number for the two fundamental modes of a beam in a periodic lattice for four neutralization factors.

\begin{tabular}{lcccc}
\hline \hline$f(\%)$ & 0 & 2.5 & 5 & 10 \\
\hline$k_{b} S$ & 0.324 & 0.331 & 0.338 & 0.351 \\
$k_{q} S$ & 0.242 & 0.252 & 0.261 & 0.278 \\
\hline \hline
\end{tabular}

and

$$
k^{*}=\sqrt{k_{0}^{2}-\frac{Q(1-f)}{\bar{R}^{2}}} .
$$

Here $k_{0}$ is the wave number of single particle oscillations in the lattice, and is also the frequency of centroid oscillations; it is related to the undepressed phase advance per lattice period $\sigma_{0}$ by $\sigma_{0}=k_{0} S$, where $S$ is the lattice period; $\sigma_{0}$ is an input parameter in our simulations. In the above expressions the mean radius $\bar{R}$ satisfies the equation

$$
k_{0}^{2} \bar{R}-\frac{K}{\bar{R}}-\frac{\varepsilon^{2}}{\bar{R}^{3}}=0,
$$

where $\varepsilon$ is the emittance.

For the cases simulated in this paper, we have $K \sim$ $1.1565 \times 10^{-3}, \varepsilon \sim 10^{-4} \mathrm{mrad}, S=0.45 \mathrm{~m}, \sigma_{0}=k_{0} S=$ $80^{\circ}$, where $\sigma_{0}$ is the undepressed phase advance. Solving (B5) gives an average radius of $\bar{R} \sim 1.13 \mathrm{~cm}$. Scaled wave numbers for the two fundamental modes are given in Table II for four values of the neutralization factor. This indicates that the wave number of both modes should increase with higher electron density. We observe this trend in our scans of frequency for the sinusoidal perturbations. For the case of amplitude modulations, which excites the breathing mode, the peak of beam loss was around $k_{b} S \sim 0.333$ for an average $f=2.5 \%$ and around $k_{b} S \sim 0.34$ for an average $f=5 \%$. For the case of shape modulation, which also triggers the breathing mode, the peak of beam loss was around $k_{b} S \sim 0.342$ for an average $f=10 \%$. For the case of "squeeze-stretch" modulation, which triggers the quadrupole mode, the peak of beam loss was around $k_{b} S \sim 0.254$ for an average $f=10 \%$.

In all cases, the observed wave numbers were significantly above the one computed for no electron density, as predicted. The observed values are remarkably close to the predictions in the case of amplitude modulation, which might be partially coincidental since the theory was in the smooth limit while the simulation involves beams departing significantly from this limit. For the case of centroid displacement of the electron density, which triggers oscillations of the beam centroid, the peak of beam loss has been observed around $k_{0} S \sim$ 0.207 for an average $f=10 \%$, below the value of $k_{0} S \sim$ 0.222 for $f=0$, in apparent contradiction with the formula (B3) which predicts larger values of $k_{0}$ for larger values of $f$. However, the assumption of a focusing effect of the electron density on the beam which led to the multiplication of the perveance by the factor $(1-f)$ does not hold in this case. An offset electron density precessing around the axis has an overall defocusing effect on the beam since it will pull the beam off axis, in effect reducing the wave number of the natural mode of oscillation.

[1] G. Budker, G. Dimov, V. Dudnikov, and V. Shamovsky, in Proceedings of the Sixth International Conference on High Energy Accelerators, Cambridge, MA, 1967 (MIT and Harvard University, Cambridge, MA, 1967), p. A103.

[2] CERN Report No. CERN-2002-001, http://wwwslap.cern.ch/collective/ecloud02/proceedings

[3] Proceedings of the 31st ICFA Advanced Beam Dynamics Workshop on Electron-Cloud Effects (ECLOUD'04), Napa, CA, 2004, edited by M. A. Furman, S. Henderson, and F. Zimmermann (Lawrence Berkeley National Laboratory Report No. LBNL-56372, 2004), http://mafurman.lbl.gov/ECLOUD04_proceedings/

[4] D. P. Grote, A. Friedman, and I. Haber, Fusion Eng. Des. 32-33, 193 (1996); http://hif.lbl.gov/theory/ WARP_summary.html

[5] E. Mahner, J. Hansen, J.-M. Laurent, and N. Madsen, Phys. Rev. ST Accel. Beams 6, 013201 (2003).

[6] A.W. Molvik, M. Kireeff Covo, F. M. Bieniosek, L. Prost, P. A. Seidl, D. Baca, A. Coorey, and A. Sakumi, Phys. Rev. ST Accel. Beams 7, 093202 (2004).

[7] J. E. Howard, Phys. Fluids 14, 2378 (1971).

[8] P. H. Stoltz, M. A. Furman, J. L. Vay, A.W. Molvik, and R. H. Cohen, Phys. Rev. ST Accel. Beams 6, 054701 (2003).

[9] G. Sabbi, A. Faltens, M. A. Leitner, A. F. Lietzke, P. A. Seidl, R. B. Meinke, J. J. Barnard, S. M. Lund, N. Martovetsky, C. Gung, J. Minervini, A. Radovinsky, and J. Schultz, in Proceedings of the Particle Accelerator Conference, Portland OR, 2003 (IEEE, Piscataway, NJ, 2003), p. 1990; http://epaper.kek.jp/ p03/INDEX.HTM

[10] J. F. Ziegler, http://www.srim.org/

[11] M. Reiser, Theory and Design of Charged Particle Beams (Wiley, New York, 1994).

[12] J. J. Barnard and S. M. Lund, in Proceedings of the U.S. Particle Accelerator School, Boulder, Colorado, 2001 (Lawrence Berkeley National Laboratory Report No. HIFAN-1106, 2001); (private communication).

[13] J.P. Boris, in Proceedings of the 4th International Conference on Numerical Simulation of Plasmas, Washington, DC, 1970 (Naval Research Laboratory, Washington, DC, 1971), U.S. Government Printing Office, stock number 0851_00059, p. 3.

[14] See, e.g., C. L. Longmire, Elementary Plasma Physics (Interscience, New York, 1963), p. 25ff.

[15] R. C. Davidson, H. Qin, P. H. Stoltz, and T.-S. F. Wang, Phys. Rev. ST Accel. Beams 2, 054401 (1999). 